\title{
La matière à l'origine du mal chez Numénius (Fr. 43 et 52 Des Places)
}

Fabienne Jourdan

\section{(2) OpenEdition}

1 Journals

Édition électronique

URL : https://journals.openedition.org/philosant/795

DOI : 10.4000/philosant.795

ISSN : 2648-2789

Éditeur

Éditions Vrin

\section{Édition imprimée}

Date de publication : 1 novembre 2014

Pagination : 185-235

ISBN : 978-2-7574-0855-1

ISSN : 1634-4561

\section{Référence électronique}

Fabienne Jourdan, «La matière à l'origine du mal chez Numénius (Fr. 43 et 52 Des Places) », Philosophie antique [En ligne], 14 | 2014, mis en ligne le 01 novembre 2018, consulté le 05 décembre 2022. URL : http://journals.openedition.org/philosant/795 ; DOI : https://doi.org/10.4000/philosant. 795

\section{(c) (i) $(9$}

Creative Commons - Attribution - Pas d'Utilisation Commerciale - Pas de Modification 4.0 International - CC BY-NC-ND 4.0

https://creativecommons.org/licenses/by-nc-nd/4.0/ 


\section{LA MATIÈRE À L'ORIGINE DU MAL CHEZ NUMÉNIUS (Fr. 43 et 52 Des Places) \\ Fabienne JOURDAN \\ Laboratoire Antiquité classique et tardive, UMR 8167, CNRS}

RÉSUMÉ. Dans son interprétation du Timée, Calcidius rapporte un exposé de Numénius sur la manière dont Pythagore, suivi par Platon, conçoit l'origine du monde. À cette occasion, Numénius identifie la matière à trois entités : la dyade indéterminée, la nécessité et l'âme mauvaise du monde évoquée dans les Lois (fr. 52 Des Places). L'article montre en quoi ces trois analogies permettent de comprendre le rôle qu'il attribue à la matière dans l'origine du mal, et ce même avant l'incarnation de l'âme, d'après les témoignages sur sa pensée fournis par Jamblique (fr. 43 Des Places) et Énée de Gaza (fr. 49 Des Places). Il analyse le contexte philosophique et exégétique de ces analogies, montrant combien la pensée de Numénius est profondément ancrée dans les cadres fournis par l'école platonicienne, tant pour ses choix herméneutiques que pour ses débats avec les autres écoles (le stö̈cisme essentiellement ici).

SUMMARY. In his interpretation of the Timaeus, Calcidius reports Numenius' account of Pythagoras' views about the origin of the universe, that later on were also taken by Plato. On this occasion, Numenius identifies matter with three other entities: the indeterminate dyad, necessity, and the evil soul of the world mentioned in the Laws (fr. 52 Des Places). The paper shows how these three analogies enable us to understand the role attributed by Numenius to matter in the origin of evil even before the incarnation of the soul, according to the testimonies on his thought given by Jamblichus (fr. 43 Des Places) and Aeneas of Gaza (fr. 49 Des Places). It analyses the philosophical and exegetical context of these analogies, showing how profoundly anchored Numenius' thought is in the framework of the Platonic school, as much for its hermeneutical choices as for its debates with the other schools (here essentially with Stoicism).

Philosophie antique, $\mathrm{n}^{\circ} 14$ (2014), 185-235 

Dans son traité sur l'âme, Jamblique rapporte deux doctrines de Numénius sur l'origine du mal. L'une la situe dans la matière, l'autre dans les corps (fr. 43 Des Places) ${ }^{1}$. Au sein de ce compte-rendu doxographique, Numénius est opposé d'une part aux platoniciens qui ne conçoivent pas la descente de l'âme dans la génération comme un mal (Plutarque et Atticus) et d'autre part à ceux qui, tout en voyant un mal dans cette descente, font de l'âme rationnelle elle-même la responsable de sa chute. Parmi les platoniciens pour qui la descente est un mal, il est ainsi rattaché au groupe qui attribue à cette chute une cause extérieure à l'âme rationnelle. Dans ce groupe, il est associé à Cronius qui incriminerait comme lui le plus souvent la matière et parfois aussi, avec Harpocration, le corps, mais il est opposé à Plotin et à Porphyre qui, de leur côté, voient la source du mal dans la nature et la vie irrationnelle. Une première tentative pour éclairer la doctrine sur la matière prêtée à Numénius dans ce fragment a consisté de notre part à la placer dans un contexte eschatologique et à la comparer à l'exposé de Macrobe sur la descente de l'âme dans la génération. L'analyse des passages du Commentaire du Songe de Scipion (I 11, 10-I 12) a permis de montrer comment Numénius pouvait, dans le domaine eschatologique et astrologique, concevoir une matière qui s'attache à l'âme de l'extérieur et l'entache de son vice, et ce avant même l'incarnation, comme le suggère en outre le fragment $49^{2}$. Le recours à Macrobe s'est toutefois avéré insuffisant, non pas parce

1. Le texte, replacé dans son contexte, est cité et traduit dans l'annexe I. Il a fait l'objet d'une première étude dans Jourdan 2013a-b, où nous expliquons cette double doctrine attribuée à Numénius. Ici, les références sont citées dans l'édition de Des Places 1973.

2. Fr. 49, extrait d'Énée de Gaza, Theophrastus, p. 12 Boissonade = PG 85, 892 b:

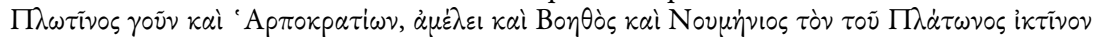

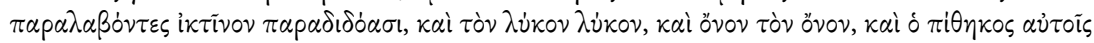

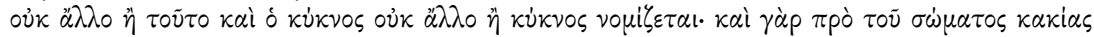

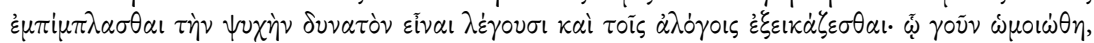

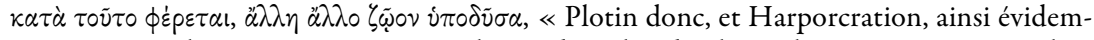
ment que Boéthus et Numénius, ayant hérité du milan de Platon, le transmettent en milan, le loup en loup, l'âne en âne; et, pour eux, le singe n'est rien d'autre que ce qu'il est et le cygne n'est qu'un cygne. Ils disent en effet que l'âme peut, avant le corps, s'emplir de perversité et se rendre semblable aux êtres dépourvus de raison. Quoi qu'il en soit, le comportement de chacune varie en fonction du vivant auquel elle s'est assimilée et dont elle a revêtu

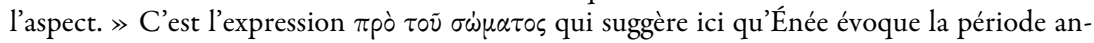


que les textes du commentaire latin sont fondés sur un réseau d'images cela correspondrait parfaitement à l'esprit de Numénius -, mais parce que leur origine numénienne est sujette à caution et qu'ils semblent parfois présenter des contradictions avec l'enseignement de l'Apaméen connu par ailleurs ${ }^{3}$.

Un second essai pour éclairer le rôle de la matière dans l'origine du mal tel qu'il est évoqué dans le fragment 43 pourrait s'appuyer sur les propos que Numénius prête lui-même à Pythagore et à Platon. L'explication relèverait cette fois du domaine cosmologique. Elle concernerait certes d'abord le rôle de la matière dans le monde et à l'égard de l'âme du monde avant de s'appliquer au rapport entre la matière et l'âme humaine, mais elle aurait l'avantage de s'appuyer sur un propos qui, bien que parvenu à travers une paraphrase de Calcidius, remonte assurément à Numénius.

Dans les paragraphes 295 à $299^{4}$ de son Commentaire du Timée, Calcidius relate la conception de l'origine du monde d'après Pythagore telle qu'elle lui est parvenue par l'intermédiaire de Numénius (fr. 52 Des Places). Dans ce cadre, Numénius identifie la matière à trois autres entités : la dyade indéterminée ${ }^{5}$ la nécessité et l'âme mauvaise du monde ${ }^{7}$. L'explicitation de ces trois identifications, ou analogies explicatives ${ }^{8}$, devrait permettre de

térieure à l'incarnation, même s'il dit plus littéralement que l'âme s'emplit de mal « avant le corps ». Comme Énée évoque Boéthus, contre lequel Porphyre a écrit son traité sur l'âme, on peut raisonnablement penser que sa source d'information est Porphyre.

3. Voir Jourdan 2013a-b.

4. Voir le texte cité et traduit dans l'annexe II. On pourrait aller jusqu'au paragraphe 300, reproduit dans la même annexe. Là Calcidius dit certes exposer les vues de Platon, mais il est évident que l'interprétation du deuxième groupe de disciples qu'il présente constitue un résumé du propos de Numénius qu'il vient de rapporter, Numénius ayant en effet harmonisé les enseignements de Pythagore et de Platon dans l'idée que le second suivait le premier. Ce résumé révèle ainsi la manière dont Calcidius comprend les positions de Numénius. Calcidius rapproche en outre ces positions d'un enseignement hébraïque étayé par une paraphrase biblique qu'il tient sans doute aussi de Numénius (sur ce passage, voir nos remarques à la note 157). Sur l'ensemble du texte, voir van Winden 1959, p. 103-123; Waszink 1962, p. 297-302 (texte et notes); Dörrie/Baltes, 1996, p. 466-471 ; Zambon 2002, p. 205-207; Moreschini 2003, p. 765-767, n. 802-826; Bakhouche 2011, p. 851-857, n. 1028-1091.

5. Fr. 52, 1. 6 Des Places. Les numéros des lignes de cette édition sont reproduits dans le texte donné dans l'annexe II.

6. Fr. 52, 1.42 et 128.

7. Fr. 52, 1. 65-67.

8. Nous employons ici le terme «identification » dans la mesure où Numénius paraît mettre ces entités sur le même plan. Mais, à strictement parler, il s'agit plutôt d'analogies explicatives. Dans cet article, la notion d'identification sera donc à comprendre en ce sens. On remarquera que Plutarque pratique la même méthode au chap. 6 du De animae procreatione à propos de l'aspect mauvais de l'âme du monde. 
comprendre comment Numénius envisage le rôle de la matière dans l'origine du mal, non seulement au niveau cosmologique, mais humain.

Quelques précisions relatives au contexte du propos rapporté par Calcidius, à la terminologie adoptée là, aux conceptions exposées et au cadre polémique, s'imposent avant toute analyse. Elles serviront à cerner les différents aspects du concept de matière à l'œuvre dans le passage.

Un mot tout d'abord sur la référence à Pythagore. Même si Numénius entend rapporter le propos de Pythagore, il étaie son exposé par des références constantes au Timée. Cette association de Pythagore et de Platon est caractéristique du platonisme de l'époque impériale. Pour parer aux dérives sceptiques de l'Académie hellénistique et retourner à un type d'interprétation dogmatique , on prétend que Platon n'aurait fait que perpétuer l'enseignement de Pythagore et que la doctrine de l'un et de l'autre, exprimée de manière voilée, exige d'être décryptée ${ }^{10}$. Telle est du moins la relation dans laquelle Numénius place les deux philosophes ${ }^{11}$. Dans ce contexte, en rapportant la pensée de Pythagore, Numénius exprime donc essentiellement sa lecture de Platon et, de fait, sa propre doctrine. Quelles sont alors ses sources? Si, concernant Platon, il renvoie ici au Timée, tout en se référant au Théétète, au Phèdre, aux Lois et sans doute aussi au Philèbe ${ }^{12}$, concernant Pythagore (qui est censé n'avoir rien écrit), il dispose sans doute du témoignage d'Aristote, des écrits néopythagoriciens circulant à son époque (pensons par exemple au Pseudo-Timée de Locres) et peut-être surtout de la tradition issue de l'ancienne Académie qu'il estime relativement fidèle à Platon, et, par voie de conséquence, au prétendu maittre de celui-ci.

Ce cadre exégétique peut permettre de mieux cerner le concept de matière à l'œuvre dans son exposé. Numénius, suivant Pythagore, pose deux principes à l'origine du monde : le dieu, associé à la monade, et la matière, associée à la dyade. Cette dernière, tant qu'elle est indéterminée, est, tout comme le dieu, inengendrée, mais dès qu'elle est déterminée, elle est considérée comme engendrée. Ces deux états correspondent à ceux que Numénius attribue à la matière : inengendrée avant la naissance du monde, et engendrée, c'est-à-dire mise en ordre par le dieu démiurge, lors de la formation du monde ${ }^{13}$. Trois traits caractérisent la matière dans cette fonction de principe originel : elle est désignée comme la mère et la nour-

9. Sur ce point, voir Frede 1987, p. 1043-1044 et le compte-rendu de Donini 2011, p. 288.

10. Voir la critique de Numénius contre l'infidélité de l'Académie à l'égard de Platon aux fragments 24-28.

11. Voir les fr. 1 et 24.

12. Sur ces références, voir $n .175$ à la traduction du texte dans l'annexe II.

13. Fr. 52, 1. 5-14. 
rice $\mathrm{du}$ monde $\mathrm{e}^{14}$; son indétermination originelle est exprimée dans sa description comme une entité fluide ${ }^{15}$, sans qualités ni formes; et son rôle de principe mauvais à l'origine des $\operatorname{maux}^{16}$ se manifeste dans son opposition au démiurge, opposition qui n'est pas seulement passive, mais active et intentionnelle ${ }^{17}$.

Cette définition constitue une transformation pythagorisante ou une appropriation, inspirée par les vues de l'ancienne Académie, du troisième genre évoqué dans le Timée, transformation inséparable du changement de terme pour évoquer ce genre. On reconnaît en effet ici l'entité à la fois spatiale et nourricière ou constitutive ${ }^{18}$ que Platon qualifie de réceptacle (vं⿰o$\delta \circ \chi \eta \dot{)}$, nourrice $(\tau \imath \theta \dot{\eta} \nu \eta)$ et mère $(\mu \dot{\eta} \tau \eta \rho)$ du devenir et dont il explique qu'elle ne peut posséder aucune forme dans la mesure où elle est l'emplacement $(\chi \dot{\omega} \rho \alpha)$ qui doit recevoir les images des formes et les qualités que sont les éléments ${ }^{19}$. Les trois autres propriétés ici attribuées à la matière, à savoir son changement d'état, son rôle de principe et plus précisément de principe du mal, et son aspect actif, peuvent s'expliquer entre autres par le cadre terminologique, polémique et exégétique où elle se trouve présentée.

1. Concernant l'idée d'un changement d'état de la matière, on pourrait remarquer d'emblée que son association à la dyade, qui est qualifiée d'indéterminée et d'indéfinie, suggère éminemment son association au genre de

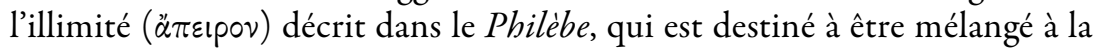
limite $\left(\pi \dot{\varepsilon}\right.$ pas) pour former le monde sensible ${ }^{20}$. Mais l'application de cette qualification plus précisément à la matière elle-même est indissociable de la terminologie à l'œuvre. Depuis qu'Aristote a identifié la $\chi \omega \dot{\omega} p \alpha$ du Timée à ce qu'il conçoit comme la ư $\lambda \eta$, la « matière ${ }^{21}$, cette entité est, dans toute la tradition postérieure à Platon ${ }^{22}$, nommée ainsi (terme traduit par silva chez Calcidius). Or la ừn n'est plus le troisième genre du Timée. Son champ définitionnel est plus large et constitue selon nous le fondement de toutes les analogies proposées par Numénius. L'influence du concept aristotélicien

14. Fr. $52,1.78,80$.

15. Fr. 52, 1. 34, 44-45.

16. Fr. $52,1.37,39,46,57-58,78,95,97,104,106,111-112,120-121$.

17. Fr. 52, 1. 94-95.

18. Sur ce sujet, voir p. ex. Brisson [1974] $1998^{3}$, p. 211, 220 ; Pradeau 1995, p. 399 ; Ferrari 2007, p. 11 n. 12 et p. 19.

19. Timée, 50c7-51a7. Chez Numénius, il n'est toutefois plus question de la dimension spatiale sans doute à cause de l'adoption du concept de ṽ $\lambda \eta$ qui ne la recouvre pas.

20. Philèbe, 23c1-24e1.

21. Voir Physique, IV, 2, 209b11-16; 209b33-210a2.

22. Le terme a pu toutefois déjà circuler dans l'Académie. Il se trouve chez Speusippe. Comme ce dernier a cependant séjourné vingt ans à l'Académie avec Aristote, il peut le lui avoir emprunté ou, à l'inverse, le lui avoir inspiré (sur cette hypothèse, voir Happ 1971, p. 221). 
sur la représentation du changement d'état initial de la matière peut alors s'expliquer ainsi. Le passage de l'état non qualifié à l'état qualifié et donc « engendré » rappelle certes l'impression des images des éléments dans le réceptacle décrite dans le Timée. Mais la «matière » telle qu'Aristote en établit le concept se prête plus essentiellement encore à cette transformation, puisque substrat du changement et des contraires d'une part ${ }^{23}$ et forme en puissance d'autre part ${ }^{24}$, elle est par définition amenée à passer de cet état d'indétermination ou d'absence de forme à la détermination qu'est, en l'occurrence, sa mise en ordre en corps du monde.

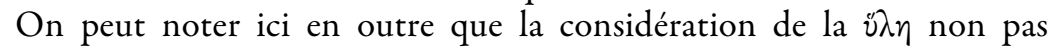
seulement comme matériau premier, mais comme l'un des principes à l'origine du monde, semble indissociable de la notion aristotélicienne de « matière » au sens de cause matérielle, c'est-à-dire de ce qui fournit les conditions de possibilité permettant la réalisation d'une forme ${ }^{25}$. Or, dans cette fonction, la « matière »n'est plus (ou plus seulement ${ }^{26}$ ) à considérer comme une «matière première » au sens « matériel »du terme $e^{27}-$ cette définition est valable essentiellement pour les objets de l'art, encore moins est-elle un emplacement où a lieu la génération ${ }^{28}$. C'est ainsi de ce concept, qui renvoie moins à un matériau premier qu'à une forme de causalité subordonnée, que Numénius hérite et qu'il utilise pour découvrir un principe du monde dans le réceptacle platonicien.

2. Le rôle de cause du mal attribué à ce deuxième principe à l'origine du monde est quant à lui un héritage du pythagorisme tel qu'il nous est connu

23. Sur cette définition de la matière, voir surtout Physique, I, 7, 190b10-191a2, où Aristote distingue la matière proprement dite, substrat du changement et réceptacle des contraires (en l'occurrence, la notion ou forme et la privation de la notion ou forme), de la

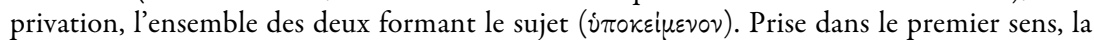
matière peut être considérée comme substance (ov̉oi $\alpha$ ). Voir Happ 1971, p. 284.

24. Sur la matière comme la forme ou l'être en puissance, voir p. ex. Physique, I, 9 , 192a27 (cf. III, 6, 206b15); Métaphysique, VIII, 1, 1042a27-28 ; VIII, 6, 1045a22-23, 1045b17-18; De anima, II, 1, 412a6-8. Prise en ce sens, la matière n'est pas substance (oủ-

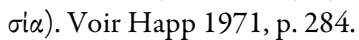

25. Sur cette définition, voir ici surtout Physique, II, 9. Sur la définition de la cause matérielle au sein de la définition des quatre causes, voir plus généralement Métaphysique, IV, 2, 1013a 24-33 et Physique, II, 3, 194b23-35.

26. La matière en tant que cause matérielle peut bien sûr être matière au sens de matière première, voir p. ex. Physique, II, 3, 195a15-17 et les passages cités dans la note précédente.

27. En fait, la matière n'est pas « matérielle » au sens où elle n'est pas corporelle (sur ce point, voir déjà Aristote, De gen. et corr. II, 1, 329a33; puis Alexandre d'Aphrodise, Commentaire du De anima, 5, 19-22 ; Plotin, Enn. II, 4 [12], 1. 15-16). Cette conception semble se faire jour dans le platonisme, héritier d'Aristote, qui précise alors que la matière n'est ni corporelle ni incorporelle, étant un corps en puissance, voir p. ex. Alcinoos, Didaskalikos, VIII, 163, 6-7 Hermann ; Hermogène, chez Tertullien, Contre Hermogène, XXXV, 2.

28. Aristote réfute précisément l'assimilation de la matière et du lieu qu'il pense lire chez Platon, voir Phys. IV, 2, 209b15, 35-36. 
par l'intermédiaire d'Aristote et que se l'est approprié la tradition platonicienne, elle aussi telle que nous la connaissons essentiellement par l'intermédiaire d'Aristote. Ce rôle est en outre souligné par le cadre polémique du propos. Dans ce passage, c'est pour s'opposer aux stoïciens que, d'après Calcidius, Numénius expose les vues de Pythagore. Il s'agit avant tout pour lui d'affirmer l'existence d'un principe originel qui explique la présence du mal. À l'inverse des stö̈ciens, en effet, Numénius considère que le mal existe réellement dans le monde ${ }^{29}$ et n'en est jamais totalement absent $t^{30}$. Le principe à son origine est selon lui la matière que ses adversaires considèrent comme neutre axiologiquement ${ }^{31}$. C'est pourquoi il a ici à cour de défendre les vues pythagoriciennes sur sa nocivité.

3. L'attribution d'un aspect actif à la matière dans son opposition malfaisante au démiurge s'explique enfin par la méthode herméneutique du platonisme de l'époque impériale qui consiste à expliquer les dialogues de Platon les uns par les autres afin de reconstruire une doctrine systématique $^{32}$. Dans le cas présent, il s'agit d'une lecture du Timée à l'aide des Lois $^{33}$ afin de rendre justice au postulat du Phèdre ${ }^{34}$ selon lequel c'est l'âme qui est principe de tout mouvement. L'âme mauvaise évoquée dans les Lois devient le principe psychique du mouvement désordonné causant le chaos précosmique dans le Timée. Or, comme il n'est visiblement aucune âme mauvaise dans ce dialogue, Numénius en découvre une dans la matière identification dont nous tenterons d'éclairer l'aspect apparemment déconcertant en renvoyant ici encore à la plasticité de la notion de matière héritée d'Aristote et peut-être déjà utilisée dans l'ancienne Académie.

Le cadre terminologique, polémique et exégétique du fragment 52 ainsi explicité, il devrait nous permettre d'expliquer comment Numénius, dans le passage transmis par Calcidius, peut identifier successivement la matière à la dyade indéterminée, à la nécessité et à l'âme mauvaise du monde. Nous essaierons ensuite de montrer en quoi chacune de ces identifications peut rendre compte de la responsabilité de la matière dans l'apparition du mal telle qu'elle est évoquée dans le fragment 43, et même de l'infection de l'âme par le mal avant l'incarnation, telle qu'elle est décrite dans le fragment 49.

29. Selon les stoïciens, il n'est de mal que moral et le sage y échappe complètement.

30. Fr. 52, 1. 44-53, 113-121. Le mal n'est pas même absent du ciel, précise-t-il, sans doute à la fois contre eux et contre les vues péripatéticiennes sur un monde sublunaire qui en serait exempt.

31. Fr. 52, 1. 2-3, 33-37.

32. Sur ce sujet, voir par ex. Donini, 1992 qui explique la méthode en général et chez Plutarque en particulier. Voir aussi Ferrari-Baldi, 2002, p. 22-24 avec la bibliographie correspondante.

33. Lois, X, 896e4-6, 897d1.

34. Phèdre, 245e5-7. 


\section{Matière et dyade}

\section{1. a. Identification entre la matière et la dyade comme cause du mal}

Une lecture superficielle de la Métaphysique (I, 6 essentiellement) pourrait donner à penser que l'association de la matière à la dyade indéterminée, conçue comme second principe de l'univers aux côtés de la monade et comme cause du mal, relève simplement de l'enseignement oral de Platon (fortement influencé par celui de Pythagore) que Numénius aurait reçu par l'intermédiaire de l'Académie. Toutefois, si la désignation de la dyade comme source du mal paraît effectivement être une doctrine discutée au sein de l'Académie, son identification à la matière semble plutôt due à une influence décisive de la présentation aristotélicienne de la doctrine platonicienne.

Reprenons le propos d'Aristote. Dans la série des contraires placés aux principes de l'univers par les pythagoriciens ${ }^{35}$, il signale que ces derniers considèrent l'Un et l'Illimité comme les principes premiers des nombres et par là de tous les êtres ${ }^{36}$, et qu'ils conçoivent l'Illimité comme la cause du $\mathrm{mal}^{37}$. Platon est censé avoir repris leur doctrine, remplaçant néanmoins la notion d'Illimité par celle de dyade ${ }^{38}$, ainsi nommée selon Aristote parce qu'elle consiste en une double entité ${ }^{39}$, « le Grand et le Petit », que Platon aurait aussi appelée l'«Inégal $»^{40}$. Sous ces trois noms, cette entité serait l'un des deux principes à l'origine des Nombres ou Idées-Nombres ${ }^{41}$ et par suite de tous les êtres, et elle serait aussi la cause du mal ${ }^{42}$. Jusque-là, Aristote rend vraisemblablement compte de discussions internes à l'Académie où étaient, comme dans le pythagorisme ancien, opposés deux principes : l'Un et le Multiple chez Speusippe ${ }^{43}$, l'Un et la dyade indéfinie chez

35. Metaph. I, 5, 986a22-b4.

36. Metaph. I, 5, 986a22 ; I, 6, 987a14-20, 987b22-26, 27.

37. Eth. Nic. II, 5, 1106b29-30.

38. Metaph. I, 6, 987b25-26.

39. Sur cette notion d'entité double, voir p. ex. Metaph. I, 5, 987a24-25; Phys. I, 9 , 192a10-12.

40. Metaph. XIV, 4, $1091 \mathrm{~b} 31$ et 35 (cf. XIV, 1, 1087b5-12).

41. Metaph. I, 6, $987 \mathrm{~b} 21$ (où le texte n'est toutefois pas sûr); XIV, 1, 1087b14-15. Nous n'entrerons pas ici dans le débat sur la notion d'idées-nombres attribuée à Platon par Aristote et renvoyons p. ex. à Cherniss 1993, p. 77-103; Richard [1986] 2005, p. 205-218.

42. Metaph. I, 6, 988b15; XIV, 4, 1091b32; Phys. I, 9, $192 \mathrm{a} 15$.

43. Voir Aristote, Metaph. XIII, 9, 1085a32-33; Jamblique, De communi mathematica scientia, 16,15. Aristote parle toutefois chez lui d'un principe analogue à la multiplicité qui n'est pas la multiplicité en elle-même. Sur le concept à l'œuvre chez Speusippe, voir Happ 1971, p. 220-240, qui le considère comme un concept de matière. 
Xénocrate ${ }^{44}$. Si Speusippe évitait de donner une valeur axiologique à ces deux principes ${ }^{45}$, Xénocrate aurait fait en revanche du second la cause du mal, s'associant par là à Platon tel qu'il est censé s'être exprimé oralement ${ }^{46}$. Toutefois, l'association du second principe à la « matière » est quant à elle loin d'être évidente chez les représentants de l'Académie. Il est en effet conçu, selon les cas, comme principe de multiplicité ou d'indétermination (de défaut de limite chez les pythagoriciens) et se voit par suite décrit comme « humide » ou «fluide » chez Speusippe ${ }^{47}$ et Xénocrate ${ }^{48}$, image reprise par Numénius ${ }^{49}$. Mais sa qualification de « matière » (vं $\lambda \eta$ ), chez les deux académiciens, semble à chaque fois due à une glose doxographique insérée dans le témoignage de leur pensée ${ }^{50}$. En outre, même dans la pensée prêtée à Platon, l'association de la dyade à la matière ne va pas de soi. Si l'on reprend le fameux passage de Métaphysique, I, 6, qui semble poser cette équivalence, on se rend compte en effet qu'Aristote présente l'Un et la Dyade comme les principes des Nombres ou Idées-Nombres ${ }^{51}$, lesquels sont ensuite au principe de toutes choses, et dit que le premier tient lieu d'ov̉oi $\alpha$ tandis que le second joue le rôle de matière. Dans l'expression $\dot{\omega} \varsigma \mu \varepsilon \dot{\nu}$ oṽv

44. Voir les témoignages d'Aétius I, 3, 8; I, 7, 18 et I, 7, 31, et leur analyse par Happ 1971, p. 249-250. Ces textes ne figurent pas dans les recueils de témoignages de Heinze 1892 et de Isnardi Parente 1982.

45. Metaph. XIV, 4, 1091b32-1092a5. Sur cette position de Speusippe, voir Happ 1971, p. 231-234 et 773-774.

46. Sur ce point, voir Happ 1971, p. 258 et 773. - Concernant la relation entre Platon et Xénocrate, nous n'entrerons pas ici dans le débat sur l'œuvre orale ou la leçon perdue de Platon. Nous rapportons simplement les propos d'Aristote. Pour un résumé de ce débat, voir Richard [1986] 2005, p. 21-47 et la préface à ce livre par P. Hadot, p. 7-15.

47. Jamblique, De communi mathematica scientia, 15, 11-14. Sur ce passage, voir p. ex. Happ 1971, p. 236.

48. Fr. 28 Heinze = 101 Isnardi Parente (Aétius, I, 3, 23, p. 288b15-18 Diels). Sur ce passage, voir p. ex. Happ 1971, p. 245 ; Isnardi Parente 1982, p. 335-337.

49. La matière est associée à l'eau dans les fragments 3 , 30 et 33 ; au lait, dans les fragments 32 et 35 . La fluidité de la matière et des corps est décrite de manière générale dans les fragments 3 et 4. Voir aussi Alcinoos, Didaskalikos, XI, 166, 27 Hermann. Sur l'association de la matière à un fluide, voir en général Decleva Caizzi 1988.

50. Concernant le témoignage de Speusippe chez Jamblique, on peut hésiter, mais il paraît évident dans le témoignage de Xénocrate (Fr. 28 Heinze $=101$ Isnardi Parente = Aétius, I, 3, 23, p. 288b15-18 Diels) que le terme ủ $\lambda \eta$ est une glose interprétative, voir Happ 1971, p. 145, n. 839. De la même façon, dans le témoignage fourni par Aétius (I, 7,

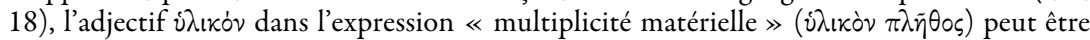
une glose. On rappellera enfin que dans le fr. 15 Heinze $(=213$ Isnardi Parente = Aétius I, 7, 30, p. 304 Diels) la dyade n'est pas associée littéralement à la matière, mais à l'âme du monde (sur ce texte, voir p. ex. Happ 1971, p. 247-248; Hager 1987, p. 73-77). Toutefois, sa qualification de « mère des dieux » peut éventuellement faire allusion à son rôle de matière ou avoir été interprétée ainsi par les successeurs de Xénocrate comme Numénius. Sur ce point, voir Jourdan 2014, n. 156.

51. Voir n. 41 avec le rappel de l'incertitude sur la lettre du texte. 


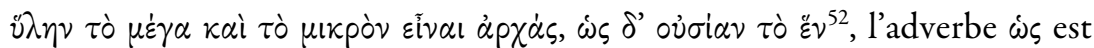
essentiel : la dyade n'est pas la matière, mais elle joue le rôle d'une matière, c'est-à-dire, dans l'esprit d'Aristote, qu'elle tient lieu à la fois de substrat sous-jacent à l'Un pour engendrer le nombre et de cause matérielle dans ce processus d'engendrement ${ }^{53}$. La matière à l'œuvre n'a plus aucun rapport avec la $\chi \dot{\omega} \rho \alpha$ du Timée et, dans la suite du texte aristotélicien ${ }^{54}$, l'expression

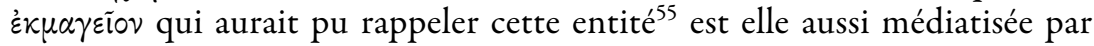
un adverbe de comparaison ( $\omega \sigma \pi \varepsilon p$ ). L'identification de la dyade à la matière est ainsi sans doute un glissement de sens simplificateur, vraisemblablement inspiré par Aristote lui-même ${ }^{56}$, et elle ne renvoie pas d'emblée, sinon de manière imagée, au matériau premier du Timée. De fait, quand, héritier de ce complexe exégétique, Numénius dit que le monde est le produit du dieu et de la matière, il ne considère pas celle-ci comme une matière première, ou du moins pas uniquement, son réseau d'analogies lui permettant de superposer les différents sens du terme.

Cette identification de la dyade à la matière, à laquelle se laisse entraîner sans doute Aristote lui-même, s'accompagne alors d'une association de la seconde elle aussi à la cause du mal. Aristote, de son côté, est gêné par un tel résultat. Pour lui, la matière est forme en puissance. Elle tend à la forme et la désire, tout comme la femelle désire le mâle et le laid le beau ${ }^{57}$. Elle ne peut donc être cause du mal, sinon par accident ${ }^{58}$, parce qu'elle accueille la privation qui est l'absence de forme ${ }^{59}$. Le mal doit donc résider dans ce deuxième aspect de la matière, la privation, qui, en tant qu'absence de forme, peut être considérée comme non-être et éventuellement comme malfaisante (kakotolóv $)^{60}$. Toutefois, les platoniciens, d'après Aristote, ne font pas cette distinction. La dyade ne correspond pas chez eux à la privation. Malgré son aspect double («Grand et Petit »), elle est totalement identi-

52. Metaph. 1, 6, 987b20-21.

53. Thémistius explicite ce processus dans son commentaire du De anima d'Aristote

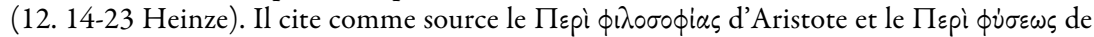
Xénocrate. D'après son interprétation de ces textes, la matière qui est présente dans les corps est alors une image (on pourrait dire aussi une manifestation) de la dyade. Voir le texte traduit en français dans Brisson 2000, p. 93-95.

54. Metaph. I, 6, 988a1.

55. Elle sert à définir la matière en Tim. 50c2.

56. Voir Metaph. XIV, 1, 1087 15, où la même doctrine est reprise de façon justement simplifiée et où cette identification a lieu.

57. Phys. I, 9, 192a13-25.

58. Phys. I, 9, 192a23-25. Sur ce refus d'Aristote d'associer matière et mal, voir Happ 1971, p. 772-774.

59. Phys. I, 9, 192a3-6, 23-25.

60. Sur ce point, Aristote n'est pas affirmatif. Il rapporte avant tout la doctrine des platoniciens sur la dyade. Cela ne signifie pas qu'il conçoive effectivement la privation comme cause du mal. 
fiée par eux à la matière. Les deux entités ne reflètent pas deux aspects. Toutes deux sont donc, dans le système platonicien ainsi présenté, non-être et mal. En faisant ainsi aux platoniciens ce reproche d'avoir conçu la matière tout entière, identifiée à la dyade, comme mauvaise, Aristote paraît avoir scellé dans l'esprit des traditions postérieures l'idée que la dyade platonicienne était conçue comme une matière mauvaise ${ }^{61}$. Il nous semble du moins que c'est de cette lecture aristotélicienne des principes de l'ancienne Académie qu'hérite Numénius quand il associe la matière avec la dyade conçue comme second principe du monde et cause du mal.

1. b. La dyade comme cause du mal qui infecte l'âme avant l'incarnation

Le processus d'identification de la matière avec la dyade étant à présent éclairé, reste à expliquer plus exactement en quoi elle permet de faire de l'entité ainsi obtenue une cause du mal.

D'après les sources platoniciennes et pythagoriciennes, la dyade apparaît comme un principe de multiplicité et d'illimitation ou d'indétermination, deux notions contraires à celles d'unité et de limite conçues comme le bien et l'ordre apportés précisément par l'action démiurgique dans le Timée. Le concept même de dyade, explicité par celui, double, de « Grand et Petit », traduit peut-être précisément cette idée d'indétermination en évoquant l'image d'une oscillation permanente entre deux pôles contraires, comme il s'en produit dans le monde sensible. Il est de fait à rattacher au principe de l'illimité ( $\ddot{\pi} \pi \varepsilon ı \rho \vee v)$ décrit dans le Philèbe ${ }^{62}$.

À cela s'ajoute que la dyade est en elle-même conçue comme une puissance de multiplication, de séparation et de division ${ }^{63}$, trois processus eux aussi contraires au bien que représente l'union dans l'esprit philosophique de l'époque ${ }^{64}$.

On peut noter enfin qu'Aristote assimile encore la dyade à l'Inégal ${ }^{65}$. Or le lecteur du Timée ne peut s'empêcher de voir dans cette expression une allusion à l'inégalité présentée là comme cause de l'absence d'uniformité et

61. Sur ce point, voir p. ex. Brisson [1974] 1998³, p. 227-232.

62. Sur ce lien, voir Reale 1984, p. 475-481.

63. Cette conception est déjà présente chez Speusippe à propos du multiple : voir Jamblique, De communi mathematica scientia, 15, 11-12, 18-20, 24-25; 17, 17 (le passage est analysé par Happ 1971, p. 235-236). On la retrouve, explicitée, chez Plutarque, De def. orac. 35, 429A-D.

64. Certes, en réalité, au niveau intelligible, ces processus servent à la distinction des idées et à leur participation réciproque. La dyade n'agit comme principe du mal qu'au niveau cosmique et humain, là où seulement le mal peut régner, d'après Platon lui-même (Theaet. 176a-b). Mais cette fonction de division et de multiplication est paradigmatique du mal qui règne dans le monde.

65. Metaph. XIV, 4, $1091 \mathrm{~b} 31$ et 35 (cf. XIV, 1, 1087b5-12). Le terme grec est äviбov. Voir n. 40 . 
par là du mouvement désordonné dans le chaos précosmique ${ }^{66}$. Dans le cadre du Timée, certes, cette inégalité s'explique très concrètement par la transformation perpétuelle des éléments les uns dans les autres. Elle n'y est pas présentée comme un concept susceptible d'être assimilé à l'un des principes de l'univers à l'image de la dyade. Mais si Platon a effectivement associé la dyade à l'Inégal, comme le prétend Aristote, il n'est pas impossible qu'il ait voulu ici faire allusion à cette théorie. Auquel cas, la dyade-inégalité aurait pu être réellement conçue chez lui comme la cause du mouvement désordonné à l'origine du mal dans le contexte cosmologique. C'est du moins la lecture qu'Eudème, à la suite d'Aristote, fait du passage ${ }^{67}$. Sa justesse est invérifiable, mais il n'en demeure pas moins que si l'association de la dyade à l'Inégal n'avait pas chez Platon lui-même contribué à doter la première des caractéristiques du second pour la définir comme une cause positive du mal, elle accentue du moins chez ses successeurs l'aspect négatif attaché à la dyade.

Toutes ces qualifications de la dyade concourent à expliquer en quoi, lorsqu'il associe la matière à la dyade, Numénius donne réellement à son lecteur une clef pour comprendre le rôle malfaisant qu'il attribue à la matière. Cette association permet alors de mieux saisir le propos du fragment 43. D'une part la source du mal ainsi conçue paraît en effet d'abord complètement étrangère à tout principe psychique. Elle peut même, d'autre part, être envisagée comme cause de l'incarnation considérée dans ce fragment comme un mal. Dans le contexte du Timée qui est à l'œuvre ici, Numénius pourrait effectivement avoir pensé que, l'âme étant une entité par nature destinée à s'incarner ${ }^{68}$, cette incarnation ne peut avoir lieu sans

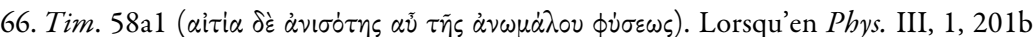
20-21, Aristote évoque certains philosophes qui ont défini le mouvement comme $\dot{\tau} \tau$ pót

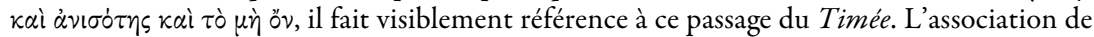
la dyade au chaos précosmique est prêtée aussi à Platon par Hermodore et Eudème d'après deux passages de Simplicius (In Phys. p. 248 5-16, 430, 34-431, 16 Diels ; voir Reale 1984, p. 552-553).

67. Concernant Aristote, voir la note précédente. Le passage d'Eudème est quant à lui

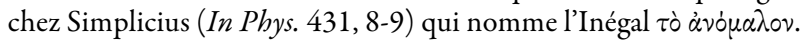

68. Numénius peut s'appuyer sur le Timée. Au plan cosmique, on peut rappeler la réflexion du démiurge du Timée à cet égard. Le monde ne peut être bon que s'il est doué d'un intellect (voṽs); or un intellect ne peut être présent à quoi que ce soit sans une âme; il faut donc le placer dans une âme et placer ensuite cette âme dans le corps du monde pour qu'il soit concrètement dans le monde (30b1-5) - réflexion qui implique l'« incarnation » de l'âme cosmique. Concernant l'homme, le Timée (42a2) dit que la première incarnation se fait $\dot{\xi} \xi \dot{\alpha} \nu \dot{\alpha} \gamma \kappa \eta \varsigma$ et que le démiurge l'organise d'une part en informant les âmes rationnelles qu'il a forgées du sort qui les attend, et d'autre part en ordonnant aux dieux secondaires de leur fabriquer une âme et un corps mortels qui vont justement permettre cette incarnation. 
l'intervention du deuxième principe constitutif du monde, la dyade ${ }^{69}$. L'association proposée rend même aussi compte du propos du fragment 49 qui précise que le mal contamine l'âme avant l'incarnation. Étant au fondement même du processus, la dyade agit sur l'âme avant qu'elle ne s'incarne $^{70}$, non pas (ou pas seulement) dans un sens chronologique, mais dans un sens causal. Enfin, par ce même statut, joint à sa définition comme principe de séparation et de multiplication, la dyade peut expliquer l'apparition de l'âme irrationnelle qui semble, chez Numénius, venir s'ajouter à l'âme rationnelle ${ }^{71}$. Porphyre évoque du moins chez lui l'existence de deux âmes, l'une rationnelle, l'autre irrationnelle ${ }^{72}$, et l'on peut supposer que, toujours suivant le Timée, Numénius concevait l'apparition de l'âme irrationnelle au moment de l'incarnation. Or cette âme semble être par excellence chez lui le lieu où se manifeste le mal provoqué par le plaisir ${ }^{73}$.

\section{Matière et nécessité}

La deuxième identification de la matière susceptible d'expliquer son rôle dans l'apparition du mal intervient à la fin du fragment 52 (1.121-129),

69. On pourrait dire ainsi que le raisonnement du démiurge est la cause première et intelligible du processus, tandis que la dyade en est la cause matérielle. Ajoutons que la dyade n'agit sans doute pas avant le monde sensible, c'est-à-dire qu'elle n'exerce pas ses effets dans la sphère divine. En revanche, elle agit à tous les niveaux du monde sensible, donc dès la formation même de l'âme (Macrobe dirait dès la descente de l'âme à travers les sphères).

70. Au niveau cosmique, cela expliquerait l'existence d'une âme mauvaise du monde.

71. Nous pensons à un ajout plutôt qu'à une division d'une âme unique en deux âmes. Cette hypothèse se fonde sur deux raisons. Numénius, d'une part, ne cesse d'interpréter le Timée, où il est précisément question de l'ajout de l'âme mortelle à l'âme immortelle. Dans les fragments et témoignages qui nous sont parvenus, d'autre part, il n'évoque la division qu'à propos du démiurge et jamais de l'âme (sur ce sujet, voir Jourdan 2014).

72. Fr. 44 (Porphyre, Sur les puissances de l'âme, chez Stobée, I, 49a25, p. 350, 25-351, 1

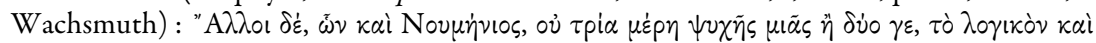

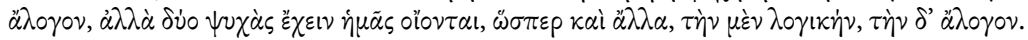

73. Cette hypothèse se fonde sur les remarques suivantes. Dans le Timée, l'âme mortelle est non seulement par essence destinée à être le lieu d'apparition et de transmission des sensations et passions, mais elle est plus précisément celui où naît le plaisir, considéré comme

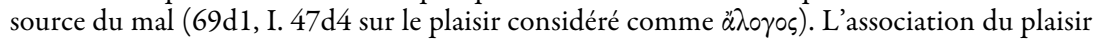
ainsi conçu à l'âme irrationnelle se retrouve chez Numénius. D'après le témoignage de Porphyre (fr. 32, 1. 9-11, De antro nymph. 28, p. 75, 11-76, 1 Nauck), qui recourt à lui pour rapporter les positions de Pythagore, Numénius aurait d'une part expliqué l'attirance des âmes dans la génération par l'effet du plaisir $\left(\delta \imath^{\prime} \eta \dot{\gamma} \delta 0 v \tilde{\xi} s\right)$. Or l'incarnation aurait été par lui considérée comme un mal (fr. 48). D'autre part, il aurait évoqué dans ce cadre les âmes déjà « habituées à la génération 》 ou «s'étant préoccupées d'elle 》 (selon la traduction adoptée

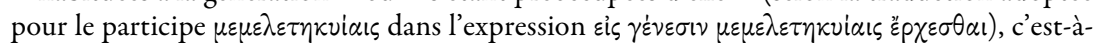
dire les âmes ayant déjà été incarnées ou du moins ayant déjà été d'une certaine façon associées à l'âme irrationnelle (ne serait-ce que dans la phase précédant l'incarnation), ce qui signifie bien qu'il associe le plaisir à cette âme. 
lorsque Numénius invite, au terme d'un raisonnement par abstraction ${ }^{74}$, à définir la matière comme l'équivalent de la nécessité.

\section{2. a. L'identification de la matière avec la nécessité}

Cette identification peut avoir été inspirée par une lecture du Timée. Elle a toutefois sans doute été scellée par l'appropriation aristotélicienne des entités à l'œuvre dans ce dialogue. Comment, en effet, un lecteur de Platon qui veut remonter à Pythagore et connaît la tradition postérieure au fondateur de l'Académie parvient-il à une telle identification ? Du pythagorisme, avons-nous dit, il veut tout d'abord retenir l'idée que le monde est le produit de deux principes. Dans le Timée, il ne trouve pas ces deux principes comme tels (Platon refuse de se prononcer sur ce sujet) ${ }^{75}$, mais deux causes ou plutôt deux types de causes à l'origine de la formation du monde $\mathrm{e}^{76}$ (Timée de Locres souligne également ce point ${ }^{77}$ ). Les unes, orientées vers l'avènement du meilleur ${ }^{78}$, relèvent de l'intellect et sont divines, les autres, dépourvues d'orientation propre, relèvent de la nécessité et sont, pourraiton dire dans un langage moderne, mécaniques ${ }^{79}$. Elles constituent en effet l'ensemble des mécanismes qu'on tendrait à désigner, en raison de leur régularité, comme « lois naturelles », si cette expression n'occultait pas la dimension hasardeuse qui les caractérise ${ }^{80}$ dans la mesure où leurs résultats ne répondent à aucune fin et que, livrés à eux-mêmes, ils produisent le plus grand désordre ${ }^{81}$. Ces mécanismes ainsi compris sont entre autres ${ }^{82}$ les sui-

74. Un tel raisonnement est préparé par le discours de Platon sur la $\chi \dot{\omega} p \alpha$, qui, ne pouvant être identifiée à aucun des élements et étant complètement dépourvue de qualité et de forme, n'est saisissable ni par la raison ni par les sens, mais seulement au terme d'un raisonnement bâtard (Tim. 51a5-b1, 52b2-3) ; et par celui d'Aristote sur le lieu (Phys. IV, 1, 209a $1-22)$.

75. Timée, en effet, ne veut pas prendre position sur la question de savoir s'il y a une ou plusieurs ápxai de l'univers (Tim. 48c3-4).

76. Tim. 48a1, 68e6-69a5.

77. Sur la nature du monde et sur l'âme, 1, 93a5-6 Marg.

78. Tim. 46e3-4. Même s'il n'y a pas de cause finale à proprement parler dans le Timée, on ne peut que constater que tout ce que fait le démiurge vise à l'avènement du meilleur.

79. Tim. 46e5-6. La notion de mécanisme est moderne, mais le terme peut rendre l'idée de Platon à condition de l'utiliser avec des réserves et de rappeler que les mécanismes en question, lorsqu'ils ne sont pas subordonnés à une intelligence qui les ordonne, produisent leurs effets au hasard. Sur ce point, voir les réserves de Taylor 1928, p. 300-301, reprises par Cornford [1937] 1966, p. 164-166, le débat étant aussi résumé par Reale 1984, p. 531-534.

80. Sur cette expression, voir de même les réserves de Taylor, reprises par Cornford [1937] 1966, p. 164 et plus généralement p. 164-176.

81. Sur ce point, voir l'explication de Reale 1984, p. 533 et l'image éclairante de la machine à laver qui, malgré ses mouvements réguliers et programmés, laisse les vêtements dans le plus grand désordre, chez Johansen 2004, p. 94.

82. Nous résumons. Si, en effet, la forme et le fonctionnement du corps sont l'objet de la réflexion du démiurge et des dieux secondaires en vue toujours du meilleur (72e-81e), les propriétés des éléments (58a-62c, cf. 46a-c à propos du principe de la réflexion), les méca- 
vants : rapprochement du semblable vers le semblable, mouvement du léger vers le haut, du lourd vers le bas, phénomènes liés à la chaleur, au froid, à la condensation et à la raréfaction ${ }^{83}$. Ces causes du deuxième type sont conçues de deux manières dans le Timée. Lorsqu'elles sont utilisées en vue de la mise en ordre du monde par le démiurge qui représente l'intellect, elles sont nommées « causes collaboratrices » $(\sigma u v \alpha i \tau / \alpha)^{84}$ et présentées comme se soumettant au processus de formation du monde tel qu'il est régi par l'intellect $^{85}$; lorsqu'elles sont considérées en elles-mêmes ${ }^{86}$, en l'absence de relation au dieu démiurge et donc de l'intellect, ce qui ressort est leur défaut de raison ${ }^{87}$ et donc d'orientation, qui vaut à la nécessité à l'état pur ${ }^{88}$ sa qualification de $\ll$ cause errante ${ }^{89}$. Dans les deux cas, ces causes se caractérisent par un certain type de mouvement. Timée dit en effet que, par nature, la

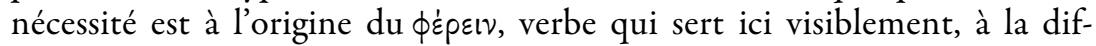
férence de kiveı à l'actif, à exprimer un mouvement d'origine contrainte ${ }^{90}$. Il précise du moins ici que de la nécessité relève tout ce qui reçoit son mou-

nismes de fonctionnement du corps eux-mêmes (sensations, impressions, exercice des cinq sens : 45b-46a, 64a-68e), tout comme ceux de ses maladies (81e-86b) relèvent quant à eux de la nécessité. L'action conjointe des deux types de causes est précisément décrite en $72 \mathrm{e}-$ 81e lorsque sont évoqués le dessein divin dans la construction du corps et l'utilisation, pour le réaliser, des propriétés naturelles des éléments avec lesquels la divinité doit composer.

83. Sur ce dernier point, voir Tim. 46d1-2.

84. Tim. 46c7, d1, 76d6.

85. Tim. 48a3-5. Cet aspect peut faire penser à ce qu'Aristote concevra comme la nécessité envisagée sous son aspect hypothétique, c'est-à-dire comme condition ou moyen en vue

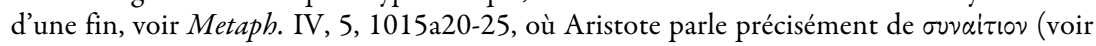
aussi Phys. II, 9). Sur ce point, voir Cornford [1937] 1966, p. 173-174; Happ 1971, p. 717 , 721-728.

86. Cette fois, on peut penser à la nécessité simple d'Aristote, par laquelle le feu ne peut faire que brûler, voir Metaph. IV, 5, 1015a33-35, Phys. II, 8. Voir Cornford [1937] 1966, p. 173-174; Happ 1971, p. 715, 719-720. Ces deux types de nécessités chez Aristote sont distingués en Part. an. I, 1, 642a31-36; Gen. an. IV, 3, 767b8-15 et V, 1, 778b16-19, voir Happ 1971, p. 729-730.

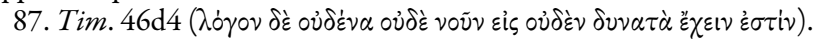

88. En elle-même, toutefois, la nécessité est susceptible de recevoir un ordre puisqu'elle peut se soumettre au démiurge, voir Reale 1984, p. 534.

89. Tim. 48a7 ( $\pi \lambda \alpha \nu \omega \mu \varepsilon \dot{\nu} \eta \alpha i \tau i \alpha)$. L'expression peut aussi avoir une connotation astronomique et faire allusion aux mouvements irréguliers des planètes. Sur ce point, voir les réflexions de Burnet [1914] 1957, p. 345-346; Cornford [1937] 1966, p. 161 ; Taylor 1928, p. 703 , n. 20 ; Reale 1984 , p. 535.

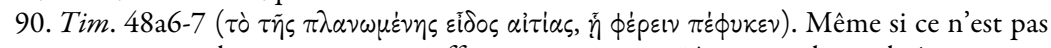

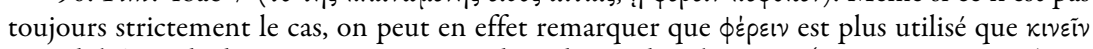
quand il s'agit de décrire un mouvement désordonné dans le Timée (voir encore p. ex. 43a7, e3, 52a5, c3, e6). D'autre part, lorsqu'il est utilisé, kıvยĩ apparaît essentiellement au passif, pour évoquer un mouvement provoqué par autre chose, un mouvement qui est donc réactif ou pris dans une série de mouvements se transmettant entre eux (voir p. ex. 46e1-2), ce qui le rend synonyme de $\phi^{\dot{\varepsilon}} \mathrm{p} \varepsilon \mathrm{v}$. 
vement d'un autre être déjà en mouvement et transmet ensuite ce mouvement à d'autres.

Comment alors cette «nécessité », qui désigne donc essentiellement un processus, peut-elle avoir été associée à la matière ? Si l'on reste dans le cadre du Timée et que l'on conçoit que la notion de matière, dans le fragment 52, renvoie à ce qui y représente le réceptacle spatial et nourricier du devenir, on peut proposer l'explication suivante. Timée introduit le réceptacle précisément au moment où il commence la deuxième partie de son discours consacrée à ce qui résulte de la nécessité ${ }^{91}$. En outre, ce réceptacle s'avère être le lieu où se manifeste la série de mouvements mécaniques qui caractérisent la nécessité. Le rapprochement entre les deux notions ainsi préparé, il pouvait ensuite être confirmé par l'observation du mouvement produit par l'entrée des éléments ${ }^{92}$ dans le réceptacle ${ }^{93}$, et plus exactement du mouvement qui a lieu sous le règne hypothétique de la nécessité pure, lorsque, avant la formation du monde, les éléments sont envisagés comme étant à l'état de « traces » d'eux-mêmes ${ }^{94}$, parce que le démiurge ne leur a pas encore conféré la structure géométrique qui les constitue. Ce moment se caractérise par un état de mouvement chaotique. Il est dû au fait que, soumis $(\pi \dot{\alpha} \sigma \chi 0 \nu \sigma \alpha)$ aux « affections » $(\pi \dot{\alpha} \theta \eta)$ produites par l'entrée des éléments encore dépourvus de toute régularité, le réceptacle est par eux mis en mouvement et, en retour, leur transmet un mouvement, ce qui engendre un processus de «secousses » réciproques ${ }^{95}$. Le phénomène qui a lieu ici est exactement celui qui définit la nécessité, à savoir la transmission d'un mouvement d'un mobile à l'autre. Il a donc pu suggérer une association plus étroite entre la nécessité et le réceptacle. Deux arguments ont pu étayer une telle interprétation. D'une part, dans ce contexte, Platon ne nomme aucun moteur ${ }^{96}$. Il n'évoque pas une âme précosmique qui provoquerait ce mouve-

91. Voir Tim. $47 \mathrm{e} 4$ où apparaît la précision sur le deuxième type de cause et $49 \mathrm{a} 1$ où est introduit le discours sur le troisième genre.

92. Platon ne tranche pas à ce niveau la question de savoir s'il s'agit des éléments euxmêmes ou d'images des éléments (51b6). Disons simplement que les éléments s'avèrent être des parties qualifiées du réceptacle $(51 \mathrm{~b} 2-6,52 \mathrm{~d} 2-4$, sur cette notion, voir p. ex. Ferrari 2007, p. 14-15), et qu'ils sont définis ensuite chacun par une structure géométrique précise (53c1-56c6) qui suggère qu'il n'y a pas d'idées des éléments à proprement parler.

93. Tim. $56 \mathrm{c} 6-57 \mathrm{c} 8$. C'est à ce mouvement de transformation réciproque des éléments les uns dans les autres au sein de la $\chi \dot{\omega} p \alpha$ que renvoie explicitement Numénius dans le fragment 52, 1. 122-125.

94. "I $\chi$ ข, Tim. 53b2.

95. Tim. 52d4-53b7.

96. La nécessité d'un moteur est évoquée en 57e3-5, mais, dans le cadre de la description du chaos précosmique, aucune entité ne joue ce rôle. Il est seulement question d'une réaction réciproque. 
ment. Celui-ci semble relever d'une simple réaction en chaîne, du $\phi \dot{e} p \varepsilon \iota^{97}$ naturel à la nécessité. D’autre part, le passage peut à la limite laisser penser que c'est le réceptacle ou les éléments qui causent le mouvement. L'un et les autres se trouvent en effet tour à tour sujets de verbes de mouvement et de changement ${ }^{98}$ à l'actif ( $\left.\sigma \varepsilon i \varepsilon l v, ~ o ́ p i \zeta \varepsilon l v, ~ \sigma u v \omega \theta \varepsilon i v\right)$. Cette description a donc pu confirmer l'intuition qu'un principe «matériel » et non psychique, dans cette situation particulière, était à l'origine du mouvement. Or, comme les éléments ne sont rien d'autre que des parties qualifiées du réceptacle ${ }^{99}$, celui-ci pouvait être ultimement considéré comme la cause du mouvement à l'œuvre. Certes, tel n'est pas le propos de Platon, puisque, dans le passage, ces actions de mise en mouvement exprimées par des verbes à l'actif ne sont en réalité que des réactions à des mouvements antérieurs ${ }^{100}$. Platon ne propose en outre jamais, dans le Timée, d'associer nécessité et réceptacle en une seule entité. Mais son propos a néanmoins pu fournir à un lecteur tenté d'opérer une telle association de quoi la nourrir ${ }^{101}$.

La cause précise que Timée donne par ailleurs à ce mouvement mécanique aurait même pu confirmer cette interprétation. Nous avons vu en effet qu'il la désigne comme l'absence d'uniformité, laquelle est due à l'inégalité $^{102}$. Cette notion renvoie au changement incessant des éléments les uns dans les autres, décrit comme un combat où certains éléments se révèlent plus puissants que leurs adversaires qu'ils dissolvent ${ }^{103}$. C'est ce phénomène précis qu'évoque la notion d'inégalité. Or ce combat est destiné à durer indéfiniment. La configuration sphérique de l'univers empêche en effet les éléments de se stabiliser en quatre masses uniformes composées chacune d'un seul type d'éléments et par là de s'immobiliser ${ }^{104}$. D'où l'absence d'uniformité et le mouvement perpétuel ${ }^{105}$. Si des interprètes

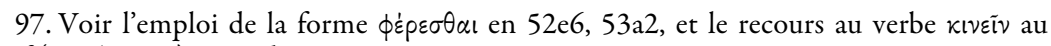
passif $(52 \mathrm{e} 4,53 \mathrm{a} 3)$, pour décrire une pure réaction.

98. Le changement étant une sorte de mouvement.

99. Voir n. 92.

100. Cela est net en 53a4-7, où l'action apparente du réceptacle est purement réactive. Platon recourt d'ailleurs à une image, puisqu'il dit que la $\chi \dot{\omega} \rho \alpha$ est comme un crible qui pro-

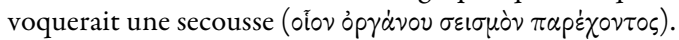

101. C'est souvent l'interprétation moderne du Timée, voir p. ex. Reale 1984, p. 549550, qui explique que réceptacle, nécessité, espace et mouvement chaotique ne sont pas des entités séparées chez Platon, mais quatre aspects d'une même entité. Cette lecture se justifie assurément, mais elle relève toujours de la reconstruction exégétique d'un système qui aurait été celui de Platon.

102. Tim. 58a1.

103. Tim. 56c7-57c6. Sans cette inégalité, chaque élément resterait l'élément qu'il est devenu et, dans ce cas, il n'y aurait plus de changement des éléments les uns dans les autres et donc plus de mouvement.

104. Tim. 57a3-5.

105. Voir aussi la suite, Tim. 58a2-c4. 
ultérieurs ont voulu trouver une allusion à l'Inégal et par suite à la dyade dans ce passage du Timée (et Numénius a pu compter parmi eux), ils ont également pu associer l'inégalité évoquée là au phénomène à la fois purement mécanique et corporel qui est littéralement décrit, et ainsi voir également dans cette inégalité un aspect de la nécessité et de la matière. Tel serait du moins le raisonnement qu'aurait pu faire Numénius s'il appuyait son interprétation sur la seule lecture du Timée.

Toutefois, comme Platon lui-même n'opère pas ces associations dans son texte, un tel raisonnement semble davantage émaner de la volonté d'y retrouver et donc d'y introduire cette interprétation elle-même. Ici encore, la pensée et la terminologie d'Aristote ont pu jouer un rôle déterminant. Dans un passage où, contre les matérialistes, le Stagirite, à l'instar de Timée lui-même ${ }^{106}$ (ainsi que du Socrate du Phédon ${ }^{107}$ ), distingue deux types de causes, celles, nécessaires, dues à de simples mécanismes, et celles orientées vers une finalité, il considère les premières comme les conditions sine quibus non de la réalisation de l'objet ou du phénomène naturel et les compare au matériau premier des objets de l'art (par exemple les tuiles et les pierres d'une maison $)^{108}$. Définissant alors le nécessaire comme ce qui concerne la matière ${ }^{109}$ des choses naturelles par opposition à sa fin qui concerne leur définition et essence, il scelle l'association entre matière et nécessité. Cette association est confirmée dans la définition de la nécessité donnée à la fin de ce chapitre de la Physique. Aristote fait d'elle « ce qui est appelé comme la matière [des choses naturelles] et les mouvements de celle-ci ». La notion de mouvement rappelle évidemment les processus mécaniques qui se manifestent dans le réceptacle du Timée et qui sont ici ceux que les matérialistes considèrent comme les seules causes à l'œuvre dans la nature. $\mathrm{Ce}$ faisant, néanmoins, Aristote n'opère pas une identification entre la nécessité et la matière conçue comme le réceptacle nourricier et spatial du Timée, ni même comme une matière première. Lorsqu'il conclut que le nécessaire dans les phénomènes naturels est ce qui est « appelé comme leur matière », il se réfere à sa notion de cause matérielle qui ici renvoie à l'ensemble des conditions indispensables à la réalisation du phénomène. Le concept ne désigne rien de matériel à proprement parler, et la définition de la nécessité à laquelle il contribue est présentée comme une comparaison. C'est lorsque le terme $u_{\lambda} \eta$ perd définitivement toute connotation matérielle pour embrasser pleinement le sens qu'Aristote lui-même lui donne, que l'identi-

106. Tim. $46 \mathrm{~d} 1-3$.

107. Phaed.98c-d. Cf. Leg. X, 889b-c.

108. Voir l'ensemble du chapitre II, 9 de la Physique.

109. Phys. II, 9, 200a13. Sur cette possibilité de traduction de la préposition દ̇v dans l'ex-

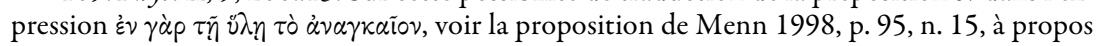
de l'expression $\dot{\varepsilon} \tau \tau \tilde{\eta} \psi u x \tilde{\eta}$ en De an. III, 5, 430a13-14. 
fication entre les deux concepts qu'il prépare ainsi peut être pleinement réalisée. Numénius pourrait témoigner d'une telle évolution interprétative $^{110}$. Elle a en effet sans doute eu lieu avant lui. On pourrait imaginer qu'elle a déjà été réalisée au sein de l'Académie ancienne, si ses représentants ont eu recours au concept de matière tel qu'Aristote le présente ${ }^{111}$ (voire en hérite ${ }^{112}$ ), ou du moins au sein des doctrines de cette Académie telles qu'elles sont ou croient être transmises, par un Plutarque ${ }^{113}$ par exemple, qui connaît bien Aristote ${ }^{114}$, ou par les néo-pythagoriciens ${ }^{115}$, qui ont eux aussi parfois recouru à ce dernier ${ }^{116}$. Mais il paraît plus vraisemblable qu'elle émane du milieu néo-pythagoricien où puise Numénius ${ }^{117}$.

110. Elle perdure chez les interprètes modernes, voir p. ex. Reale 1984, p. 531-534.

111. Le concept a pu circuler dans l'Académie à partir d'Aristote, Speusippe ayant séjourné avec lui vingt ans à l'Académie, voir n. 22.

112. C'est la thèse de Happ 1971 (plus particulièrement p. 260-276). Mais, même s'il hérite en partie du concept, Aristote le développe lui-même ainsi.

113. Voir p. ex. sa présentation des positions de Xénocrate et de Crantor sur la formation de l'âme du monde dans le Timée, au début du De animae procreatione $(\$ 2,1012 \mathrm{D}$ $1013 \mathrm{~A})$.

114. La conception aristotélicienne de la matière paraît en effet fortement inspirer la description d'Isis assimilée à la matière dans le De Iside et Osiride. Voir p. ex. le $₫ 53,372 \mathrm{E}-\mathrm{F}$ de ce traité, à comparer avec Phys. I, 9, 192a16-25. De manière générale, sur la relation de Plutarque à Aristote, voir Donini 2004 ; Chiaradonna 2011, p. 9-13.

115. Nous avons vu que le néopythagorisme naît du désir d'interpréter Platon de manière dogmatique et en cela souhaite retrouver l'esprit originel de l'Académie, perdu pendant la période sceptique de celle-ci. Voir n. 9.

116. Sur l'utilisation d'Aristote par les néo-pythagoriciens ou platoniciens pythagorisants, voir Chiaradonna 2013, p. 16-27 et Bonazzi 2013, qui tous deux traitent du cas d'Eudore. Or il ne faut pas oublier qu'Eudore semble être précisément la source de Plutarque sur les doctrines non seulement de Pythagore, mais aussi de Xénocrate et de Crantor dans le De animae procreatione (3, 1013 B ; 16, 1019 E et 1010 C, voir Cherniss 1976, p. 165, n. c).

117. Cette identification de la matière avec la nécessité peut en effet aussi être apparue dans un milieu néopythagoricien. Elle est du moins toujours liée à une interprétation de Platon et nous avons vu que ce courant était vraisemblablement né dans un contexte de renouveau de l'exégèse platonicienne (voir n. 9). Plutarque l'attribue à certains philosophes qu'il ne nomme pas mais dénonce en De animae procreatione, $₫ 6,1014$ E, p. 188 Cherniss. Or Cherniss 1976, p. 188, n. c, suivi par Ferrari \& Baldi 2002, p. 251, n. 82, renvoie à son propos au Pseudo-Timée de Locres (Sur la nature du monde et de l'âme, I, 93a-94b Marg). L'identification est aussi prêtée à Platon en termes semblables à ceux du Pseudo-Timée par Diogène Laërce (III, 75-76) et Aétius (I, 26, 3 = Dox. gr. p. 321 A 18-19 et B 19-20). Plotin l'évoque de même comme une interprétation de Platon qui semble courante dans son traité sur l'origine du mal (Enn. I, 8 [51], 7. 4-7). Plutarque la laisse encore exprimer par Lamprias lors d'une interprétation de Platon dans le De defectu oraculorum, $₫ 47$, 435 F-436 A. Cela suggère selon nous qu'il peut lui-même l'avoir provisoirement acceptée, éventuellement dans la période où il n'était pas encore tout à fait détaché du pythagorisme, avant de la rejeter. Le De animae procreatione témoignerait ainsi de son évolution. Plutarque ne nommerait pas sa source peut-être justement parce qu'il en aurait été un moment trop proche. Il est en outre 
Il ne s'agit toutefois pas ici d'affirmer que cette association n'aurait pas pu exister avant ou sans les réflexions d'Aristote ${ }^{118}$. La lecture du Timée que nous avons proposée pouvait déjà y conduire. Mais la manière dont Aristote établit le concept de matière scelle une association qui a pu s'imposer précisément parce qu'elle dégage ce concept de sa référence unique au réceptacle du Timée. Elle permet de lui conférer un autre sens et ainsi de l'identifier à une entité plus abstraite, entre autres à celle qui incarne l'ensemble des conditions nécessaires à l'existence d'un objet ou d'un phénomène, tel qu'Aristote conçoit la matière. L'adoption de cette terminologie n'empêche d'ailleurs pas le maintien d'une allusion au réceptacle du Timée, comme le montre l'interprétation de Numénius. C'est ainsi selon nous en vertu d'une lecture du Timée inspirée par le développement ultérieur du concept de matière qu'a pu se former l'identification entre celle-ci et la nécessité telle qu'il la propose à la fin du fragment 52.

2. b. Matière, nécessité et cause du mal:

le mouvement désordonné et l'incarnation

Les étapes conduisant à l'identification entre matière et nécessité ainsi reconstituées, reste à déterminer en quoi cette identification permet d'expliquer le rôle de la matière comme origine du mal, c'est-à-dire à montrer en quoi la nécessité elle-même peut être considérée comme cette source du mal, le raisonnement s'appliquant par suite à la matière. Aristote, en effet, ne confère à aucune des deux entités cette fonction, pas plus que Platon ne la prête au deuxième type de cause ni au réceptacle. Si l'on s'en tient ici à examiner le rôle de la nécessité, dans le Timée, on voit que celle-ci se laisse persuader par le démiurge en vue de la formation du monde le meilleur possible, et Numénius n'omet pas de rappeler ce processus (1. 128-129). Le seul argument que Platon aurait pu à la limite donner aux partisans de l'idée que la cause du mal réside dans la nécessité est que, lorsqu'elle est laissée à ellemême, en l'absence du démiurge et donc de toute orientation vers le bien, la nécessité est source de désordre. Le chaos précosmique représente par excellence ce désordre, état qui n'aurait pas pu être complètement résorbé dans le monde une fois formé. Ce mouvement désordonné dont elle semble être à l'origine devait ainsi permettre de faire de la nécessité la source du mal. Dans la mesure où la matière était déjà présentée dans le Timée comme le lieu où se manifeste ce mouvement dépourvu de raison et par là producteur de désordre, en l'identifiant à la nécessité, les commentateurs

vraisemblable qu'il ait connu les positions attribuées à Pythagore telles qu'elles sont exposées par Numénius (bien entendu pas par l'intermédiaire de ce dernier).

118. Étant donné l'incertitude sur la diffusion de son œuvre, il reste difficile de savoir comment son enseignement s'est répandu dans les premiers cercles platoniciens. Sur ce sujet, voir tout récemment Chiaradonna 2011 et 2013. 
scellaient ainsi leur conception de la matière comme la véritable source du mal.

Toutefois, dans le Timée, le mouvement dont la nécessité est cause n’a rien d'une opposition positive au démiurge telle que la décrit Numénius. Il ne s'avère au contraire producteur de désordre qu'en l'absence du dieu et se soumet de suite à lui dès que celui-ci le prend en main. Cette conception d'une opposition est sans doute le fruit de la fusion de la notion de deuxième cause que représente la nécessité dans le Timée avec celle du deuxième principe tel qu'il est conçu dans le pythagorisme où nous avons vu qu'il s'oppose au premier. Or, chez Numénius, ce principe est représenté par la dyade, devenue matière après adoption de la terminologie aristotélicienne, et l'on peut considérer qu'à ses yeux la nécessité (ou deuxième type de cause) constitue la manifestation cosmique de la dyade (qui est le second principe). Quant à l'aspect positif et pas seulement passif de l'opposition à l'œuvre, il se justifie d'un côté peut-être toujours par cette fusion de la notion de deuxième cause avec celle de second principe et sans doute surtout, de l'autre, par la troisième identification qui conduit Numénius à assimiler la matière à l'âme mauvaise elle-même, faisant d'elle, cette fois, un véritable principe moteur et par là une cause positive de mouvement.

Avant d'expliciter cette troisième identification, il faut encore expliquer en quoi l'association de la matière avec la nécessité peut éclairer le propos du fragment 43 selon lequel la matière est cause de l'incarnation et par là cause du mal pour l'âme humaine ${ }^{119}$, et ce, comme le précise le fragment 49 , avant même l'incarnation.

$\mathrm{Si}$, avec Jamblique, on prête à Numénius l'idée que toute incarnation est un $\mathrm{mal}^{120}$, on peut en effet voir comment Numénius a pu penser trouver exprimée dans le Timée son idée selon laquelle le mal venait à l'âme de la nécessité et donc de la matière. Dans le texte de Platon, en effet, l'incarnation relève de la nécessité à tous les niveaux. Certes, la cause initiale et véritable est la réflexion cosmologique du démiurge. Pour être le meilleur des êtres, le monde devait être pourvu d'un intellect. Or un intellect ne pouvait être dans le monde ailleurs que dans une âme. Il fallait donc doter le monde d'une âme pour y placer l'intellect, puis placer cette âme dans le corps du monde afin que l'intellect puisse s'y trouver aussi ${ }^{121}$. Le même

119. C'est ce qu'invite à conclure Jamblique dans le fragment 43, idée reprise dans le fragment 48 , voir $n$. suivante.

120. Fr. 48, extrait de Jamblique, Sur l'âme, ap. Stobée, I, 49, \$40, 21-34, vol. I, p. 380, 6-19 Wachsmuth = Jamblique, De anima, \$29, p. 56, 25-28 Finamore-Dillon : $\langle$ Tıv่s $\delta \dot{\varepsilon}$

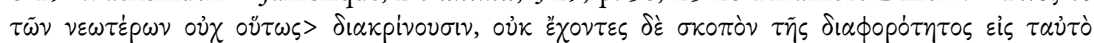

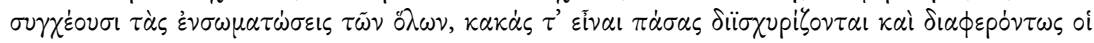

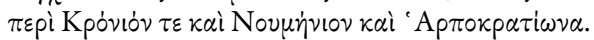

121. Tim. 30b1-5 (voir le texte cité dans la note suivante). 
raisonnement a pu valoir pour l'incarnation de l'âme humaine. Toutefois la nécessité n'intervient pas dans les deux cas. Dans celui du monde, la réflexion d'ordre intellectuel à l'origine de l'incorporation de l'âme n'est pas désignée en termes de nécessité, même si elle paraît relever d'une contrainte d'ordre « logique $»^{122}$. Elle est le fruit d'un plan réfléchi en vue de l'avènement du meilleur. C'est dans le cas de l'homme que l'incorporation tient effectivement à la nécessité, sans doute parce que son corps n'est ni immortel ni parfait, contrairement à celui du monde. Le démiurge dit en effet que c'est $\dot{c}^{\xi} \dot{\alpha} \nu \dot{\alpha} \gamma \kappa \eta \xi$, « en vertu de la nécessité », que les âmes rationnelles qu'il a fabriquées doivent s'implanter dans les corps, sans donner d'autre justification ${ }^{123}$.

En outre, la réalisation de cette implantation est confiée aux dieux formés par le démiurge, les astres, qui œuvrent certes à l'imitation de leur « père », c'est-à-dire en faisant preuve de réflexion en vue du meilleur, mais sont surtout soumis à la nécessité lorsqu'il leur faut accomplir cette association de l'âme rationnelle au corps ${ }^{124}$. En cela, l'incarnation est donc bien essentiellement le fait de la nécessité. Si Numénius considérait effectivement l'incarnation comme un mal, il pouvait donc ainsi trouver dans le Timée de quoi justifier son idée que la nécessité est la cause de ce mal qui affecte l'âme rationnelle (celle formée par le démiurge), et ce précisément de l'extérieur, puisque c'est cette cause étrangère à toute rationalité, la nécessité, qui paraît y être présentée comme à l'origine de l'incarnation. La nécessité affecte par surcroît l'âme rationnelle avant l'incarnation, non seulement parce qu'elle est à l'origine de celle-ci, mais parce qu'elle se manifeste dans toute l'œuvre des dieux secondaires destinée à la préparer contexte qui a pu ensuite nourrir l'imagerie astrale liée à ce thème chez Numénius et ses pairs ou du moins justifier cette imagerie comme étant d'origine platonicienne ${ }^{125}$.

Une dernière remarque peut alors être tirée de cette idée d'incarnation de l'âme rationnelle placée sous la gouverne des dieux secondaires. À cette

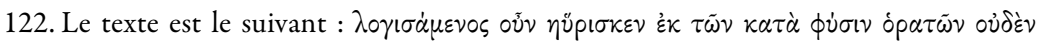

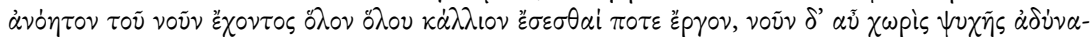

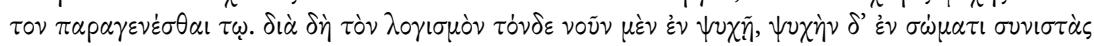

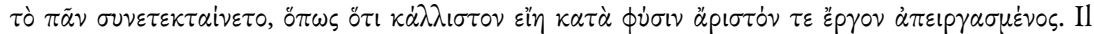

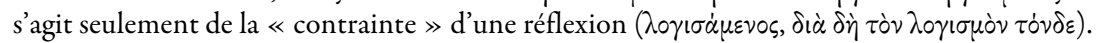

123. Tim. $42 \mathrm{~d} 6$.

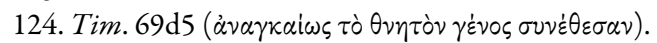

125. Sur le thème du corps astral comme véhicule de l'âme, voir en général Dodds [1933] 1963, p. 313-321; Kehl 1978. Les notions de véhicule ("̋ $\chi \mu \alpha)$ et de corps astral sont le plus souvent unies dans la littérature, mais cet usage est peut-être abusif. Sur cette imagerie présente chez Macrobe et que Numénius a éventuellement utilisée, voir Jourdan 2014 et $2013 \mathrm{a}-\mathrm{b}$. 
occasion, ces dieux fabriquent l'âme mortelle ${ }^{126}$, qu'ils font siéger dans les parties du corps différant de la tête où est située l'âme rationnelle. Or cette deuxième espèce d'âme est source des passions qui éloignent du bien et sont précisément qualifiées ici encore de fruit de la nécessité ${ }^{127}$. On peut légitimement penser que c'est concrètement cette deuxième âme qui est conçue par Numénius comme l'un des maux produits par la nécessité et qui vient s'attacher de l'extérieur à l'âme rationnelle. Dans ce contexte, Numénius a pu aussi penser trouver justifiée sa conception d'une deuxième âme humaine, l'âme irrationnelle, qu'il a sans doute considérée comme mauvaise ${ }^{128}$.

Un autre mal produit par l'incarnation est évidemment la rencontre effective avec le corps. Cette idée est liée non seulement à la considération que le mal provient de la matière en tant que nécessité source de mouvement chaotique, mais aussi cette fois en tant que matériau et substance corporelle ou origine de cette substance. Cette idée est plus exactement exprimée par la deuxième doctrine sur l'origine du mal prêtée par Jamblique à Numénius dans le fragment 43. La description de la naissance donnée dans le Timée rappelle en effet exactement l'épisode du chaos précosmique. L'union concrète de l'âme et du corps soumet la première aux flux et reflux du second qui commencent par disloquer complètement en elle tout son système de jugement ${ }^{129}$. Sont produites en outre des impressions et sensations qu'elle ne parvient pas à maîtriser et qui achèvent la dislocation ${ }^{130}$. Ce deuxième processus est dû à l'âme mortelle, qui n'est pas nommée dans le passage concerné tout simplement parce que sa formation n'a pas encore été évoquée. À ce stade, avant que l'intellect ${ }^{131}$ en elle ne la reconduise vers l'ordre, l'âme rationnelle est donc complètement soumise à la nécessité.

126. Sur cette notion qu'il faut peut-être relativiser chez Platon, voir Brisson 2011.

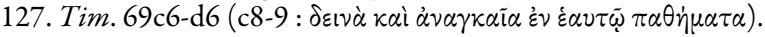

128. Le texte de Porphyre qui l'évoque n'est pas explicite sur ce caractère mauvais (voir le fr. 44 cité à la note 72). Mais c'est bien cette seconde âme qui est liée au plaisir, lequel est considéré comme la prison de l'âme rationnelle incarnée (voir le fr. 38 et les remarques à la note 73). On remarquera en outre qu'Alcinoos (Didask. XXV, 178, 23-31 Hermann, p. 50 Whittaker), à propos de l'âme irrationnelle, évoque lui aussi une deuxième espèce d'âme plutôt qu'une deuxième partie de l'âme (notion rejetée par Aristote avec lequel il tend à faire s'harmoniser la pensée de Platon). Ce faisant, il ne s'éloigne guère de la lettre du Timée, puisque les dieux inférieurs y fabriquent effectivement une autre espèce d'âme, «mortelle », qu'ils mettent en relation avec celle, divine et immortelle, fabriquée par le démiurge

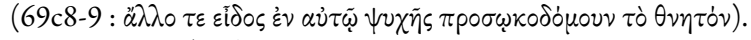

129. Tim. 43 a6-7.

130. Tim. 43b5-c7.

131. «Intellect 》 (voũs) est à entendre ici au sens non d'un organe particulier, mais de 《 rationalité » ou « réflexion rationnelle » (sur cette interprétation voir Menn 1995, dont les conclusions sont résumées dans Menn 1998, p. 87-88). Cette mise en ordre correspond à celle du monde soumis au chaos par le démiurge qui représente lui aussi l'intellect. 
C'est ainsi du fait de son rôle à tous ces stades de l'incarnation telle qu'elle est présentée par Platon lui-même que Numénius pouvait considérer la nécessité comme la cause du mal affectant l'âme rationnelle.

\section{Matière et âme mauvaise; matière auctrix et patrona de la « partie » passible de l'âme humaine d'après le fragment 52}

La troisième identification de la matière à une entité pouvant expliquer son rôle dans le mal est la plus déconcertante puisqu'elle consiste à mettre sur le même plan la matière et une âme, en l'occurrence l'âme mauvaise évoquée dans les Lois. Cette identification est due à deux lectures de Platon et, si Numénius l'accepte effectivement, c'est elle qui explique le plus aisément chez lui le lien entre matière et mal au niveau cosmique. Quant à l'explication concernant le niveau humain, elle dépend du sens que les trois identifications proposées permettent de donner à l'expression auctrix et patrona par laquelle Numénius définit le rôle de la matière à l'égard de l'âme irrationnelle, ou plutôt de ce que Calcidius nomme « la partie passible de l'âme [humaine $]^{132} \gg$.

\section{3. a. Identification de la matière à l'âme mauvaise du monde}

Malgré son caractère déconcertant, l'identification de la matière à l'âme mauvaise du monde est inspirée à Numénius par la méthode, courante dans le platonisme, qui consiste à interpréter Platon par Platon et à construire ainsi un seul système à partir des thèses exprimées dans les différents dialogues ${ }^{133}$. Le mal étant conçu, au niveau cosmique et humain, comme une forme de mouvement, en l'occurrence producteur de désordre, le commentateur de Platon a besoin de désigner une cause de mouvement telle que Platon la conçoit lui-même. Il ne peut se contenter de nommer la nécessité qui n'est pas ainsi présentée par le maître. Or, dans le Phèdre, Platon a explicitement désigné ce que devait être la cause de tout mouvement : une âme ${ }^{134}$. C'est donc une âme qui doit être ultimement conçue comme cause véritable du mouvement désordonné. Dans les Lois, Platon émet lui-même l'hypothèse de l'existence d'une âme mauvaise au niveau cosmique ${ }^{135}$. Il

132. Les lignes 70-74 du fr. 52 concernent de toute évidence l'âme humaine, à laquelle est en partie appliquée l'argumentation mise en œuvre pour l'âme du monde.

133. Sur cette méthode, voir les références données à la note 32.

134. Phaedr. 245e5-7.

135. Dans les Lois (X, 896e4-898d1), Platon se sert sans doute implicitement d'une comparaison avec l'âme humaine. Puisque, chez les hommes, on peut supposer l'existence d'une âme mauvaise, cette supposition est appliquée au monde. Or, là, l'observation des corps célestes montre que c'est l'ordre qui règne. Donc, dans le cas du monde, seule règne une âme absolument bonne. L'hypothèse d'une âme mauvaise est posée pour être immédiatement levée : même dans le cas d'une multiplicité d'âmes du monde, le texte conclut à l'excellence de chacune (898c7-8). On verra que Numénius est sans doute fidèle à Platon en ce qu'il évoque effectivement une deuxième âme du monde, mais lui retire finalement sa réa- 
suffit donc de transformer cette hypothèse en affirmation pour croire avoir trouvé chez lui la cause recherchée. Plutarque en reste à un tel raisonnement et considère alors que c'est une âme qui est à l'origine du mal dans le monde ${ }^{136}$. Numénius préfère au contraire incriminer la matière, mais la matière telle qu'il la définit, c'est-à-dire ici moins comme un matériau premier (conception qui n'est jamais exclue étant donné la référence au réceptacle du Timée), que comme le principe à l'origine du monde en tant que dyade et nécessité, principe qui tient alors précisément son aspect positif et actif de cet aspect psychique qu'il revêt.

Une deuxième exégèse de Platon a pu inspirer semblable identification. On sait par Proclus ${ }^{137}$ que Numénius a donné une interprétation de la formation de l'âme du monde dans le Timée identifiant la « substance [qui devient] divisible dans les corps $\gg^{138}$ à la dyade indéterminée. Or nous avons vu que, dans le contexte cosmologique, cette dyade est assimilée à la matière. Numénius peut donc éventuellement appartenir à la série des commentateurs auxquels Plutarque reproche d'assimiler la substance divisible (qu'il assimile quant à lui à l'âme mauvaise ou à l'aspect mauvais de l'âme ${ }^{139}$ du monde) à la matière. Plutarque qualifie alors de «corporelle » la matière en question pour mieux dénoncer ses adversaires, en l'occurrence Crantor, dont les positions n'ont en réalité rien à voir avec cette théorie ${ }^{140}$, là où ceux qui faisaient effectivement cette association n'envisageaient vraisemblablement pas la matière sous cet aspect «matériel » ou corporel $^{141}$. Des pythagoriciens ont pu être à l'origine d'une telle identification. Le Pseudo-Timée de Locres, par exemple, définit la matière par l'expression « divisible dans les corps » qui sert chez Platon à qualifier ce constituant de l'âme cosmique sur la nature duquel porte le débat ${ }^{142}$. Mais le texte qu'il nous livre ne comporte pas explicitement l'identification recherchée. C'est finalement Plutarque qui donne peut-être lui-même l'interprétation qui a pu suggérer une telle identification lorsqu'il dit, en termes très aristotéliciens, qu'il accepterait à la limite de qualifier de « matière » cette entité, si,

lité psychique puisqu'il l'identifie à la matière. En revanche, au niveau humain, il semble réellement poser l'existence d'une deuxième âme (cette fois comme dans le Timée).

136. C'est tout le raisonnement à l'œuvre dans le De animae procreatione (voir plus précisément le $\$ 6,1014 \mathrm{E}$ ).

137. Fr. 39 = Proclus, In Tim. II, 153, 17-25 Diehl.

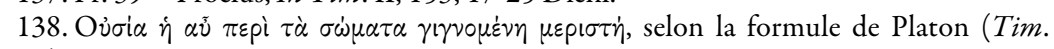

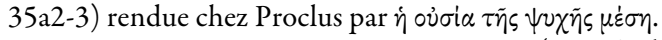

139. De animae procreatione, $\$ 7,1015 \mathrm{E}$ (p. 196 Cherniss).

140. Sur ce point, voir Cherniss 1976, p. 172-173, n. c.

141. La matière était plutôt conçue comme n'étant ni corporelle, ni incorporelle, voir n. 27.

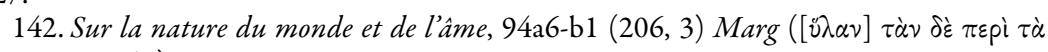
$\sigma \dot{\omega} \mu \alpha \tau \alpha \mu \varepsilon \rho \sigma \tau \dot{\alpha} \nu)$. 
par matière, on entend de manière « homonymique $»^{143}$ la nature qui sert de substrat à l'entité indivisible et qui « participe » à elle au sens où elle se laisse mettre en ordre par elle ${ }^{144}$. On retrouve ici la définition que Numénius donne de la dyade indéterminée lorsqu'il l'assimile à la matière au début du fragment 52, matière-dyade qu'il présente comme étant en soi éternelle et indéterminée, mais se laissant déterminer par la monade et par suite devenir engendrée, c'est-à-dire ordonnée. Numénius a donc pu s'approprier cette définition de la matière proposée ou reprise par Plutarque et s'inscrire dans le débat avec celui-ci, en maintenant justement que ce que le Chéronéen tenait à considérer uniquement comme une âme était en réalité aussi matière. En reprenant éventuellement à Plutarque l'idée que le constituant divisible était une âme mauvaise, Numénius obtenait ainsi encore, par une interprétation de Platon différente de celle que nous avons reconstruite dans le paragraphe précédent, l'identification de la matière à l'âme mauvaise.

Rien ne permet d'affirmer que Numénius a effectivement connu l'exégèse de Plutarque. Il a pu toutefois connaître les développements pythagorisants qui l'inspiraient. Il est du moins certain qu'il a donné sa propre lecture de la formation de l'âme du monde dans le Timée, et son identification du constituant divisible avec la dyade indéterminée a pu le porter à identifier celui-ci aussi à la matière. Le sens « homonymique » que Plutarque donne à une telle identification est parfaitement envisageable dans l'esprit de l'Apaméen tel que nous avons tenté de le reconstituer jusqu'ici. C'est pourquoi il est permis de penser qu'il s'inscrit dans la tradition exégétique avec laquelle dialogue Plutarque et qu'il n'est pas impossible qu'il lui réponde sur la notion d'âme mauvaise ${ }^{145}$.

\section{3. b. La nuisance de l'âme mauvaise au niveau cosmique}

La question de savoir comment cette identification rend compte de la considération selon laquelle la matière est responsable du mal est cette fois la plus aisée, puisque, au niveau cosmique, c'est l'âme mauvaise, en tant que

143. De animae procreatione, $\$ 21,1022 \mathrm{~F}$ (p. 214 Cherniss). Le terme « homonyme » est à prendre ici au sens de ce qui aurait non seulement même nom, mais quelque chose en commun, non pas la nature, certes, comme dans le cas des homonymes platoniciens (l'âme ne peut avoir la nature de la matière), mais la fonction, en l'occurrence celle de servir de réceptacle. Une telle acception du terme renverrait à ce qu'Aristote appelle les homonymes

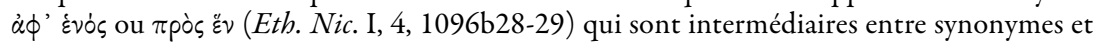
homonymes et dans lesquels la communauté de nom a sa raison d'être.

144. De animae procreatione, $\$ 21,1022$ F 2-3. On sera ici frappé par le recours au voca-

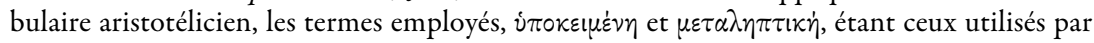
Aristote, le premier, de manière générale, pour évoquer la matière comme substrat, le second, en Phys. IV, 2, 209b12-13, pour décrire la $\chi \dot{\omega} p \alpha$ platonicienne.

145. Raison supplémentaire pour ne pas accepter la correction du texte proposée par W. Theiler (voir n. 147). 
source de mouvements désordonnés, qui est censée produire ce mal. Cette identification peut aussi rendre compte, avons-nous dit, de l'aspect positif pris par l'opposition de la dyade et de la nécessité au démiurge, et expliquer ce qui permet à Numénius de décrire en termes moraux cette opposition et ses conséquences ${ }^{146}$. Cette conclusion doit toutefois être nuancée si l'on reste fidèle à la lettre du texte. Numénius n'accepte en réalité pas totalement l'existence d'une deuxième âme du monde proprement dite. Le texte de Calcidius dit en effet que, selon lui, l'âme malfaisante est « évidemment la matière » (alteram malignam, scilicet silvam). Or, selon nous, le texte ne doit pas être modifié pour suggérer que l'apposition scilicet silvam spécifie que l'âme mauvaise est en réalité l'âme de la matière, scilicet silvae, selon la correction de W. Theiler, plutôt que la matière elle-même ${ }^{147}$. Numénius a vraisemblablement bien compris que Platon n'envisageait pas réellement l'existence d'une deuxième âme du monde et, pour lui, ce qui tient lieu de principe de mouvement désordonné ne peut être que la matière-nécessité. L'identification de celle-ci à l'âme du monde lui sert simplement à faire coïncider tous les enseignements qu'il attribue à Platon et à justifier la présence d'un mouvement propre au sein de la matière. Elle explique le rôle positif que la matière joue selon lui dans l'apparition du mal. Elle ne permet

146. Fr. 52, voir 1.41, 58, 95 (malitia), 87 (praesumptio), 97 (temeritas), 121 (piaculum), voire peut-être 85 (intemperies). On remarquera en outre que Calcidius emploie l'adjectif maligna de préférence au simple mala à propos de la matière. Cet usage est remarquable à la ligne 46, où le premier adjectif est prêté à Pythagore pour affirmer la malfaisance foncière de la matière, le second aux stoïciens qui nient qu'elle ait le moindre caractère mauvais.

147. Theiler 1955, p. 74, propose de remplacer à la ligne 67 silvam par silvae et suggère ainsi que ce n'est pas avec la matière, mais avec l'âme de la matière que Numénius identifie l'âme mauvaise (Bakhouche 2011, p. 854, n. 1057, accepte cette correction). Certes, d'après le texte retenu par les éditeurs modernes depuis Waszink 1962, p. 300, Numénius évoque plus loin l'âme de la matière (silvae anima, fr. 52, 1.92), sans doute pour mieux rendre compte de l'aspect psychique de l'opposition au démiurge. Mais ce passage a donné lieu à des hésitations puisque, dans certains manuscrits, au lieu de silvae anima, on lit silva animae, « la matière de l'âme » (voir l'apparat critique de Waszink 1962, p. 300), ce qui prouve bien que les copistes avaient parfaitement compris le raisonnement précédent de Numénius qui consistait à identifier l'âme mauvaise à la matière. Ce raisonnement nous paraît d'ailleurs parfaitement clair : Numénius semble avoir mieux interprété ce passage de Platon que ses collègues, au sens du moins où il n'a pas pensé que Platon admettait effectivement l'existence de deux âmes du monde. Platon ne peut concevoir l'existence d'une âme mauvaise au niveau cosmique (voir n. 135). Numénius dit alors que cette deuxième âme évoquée correspond en fait à la matière-nécessité, et cela lui semble si évident qu'il écrit malignam alteram, scilicet silvam, « l'autre [âme], malfaisante, c'est-à-dire évidemment la matière 》 (voir aussi la traduction de Moreschini 2003, p. 599). C'est exactement le raisonnement inverse de celui de Plutarque. 
pas de conclure qu'il croit, contrairement à Plutarque, à l'existence réelle d'une âme mauvaise du monde ${ }^{148}$.

Si l'identification de la matière à l'âme mauvaise du monde permet du moins de comprendre aisément la présence active du mal au niveau cosmique, la question est en revanche plus difficile à résoudre au niveau humain. Certes, il est aisé de voir que, là, le mal se manifeste dans les passions de l'âme irrationnelle et le Timée est explicite à ce sujet. Mais qu'est-ce qui, dans l'âme mauvaise du monde assimilée à la matière, peut expliquer ce mal qui caractérise l'âme humaine ? La réponse la plus simple serait de supposer que Numénius considère l'âme irrationnelle de l'homme comme issue de cette « âme » cosmique mauvaise, qu'elle en soit un produit, en constitue une partie ou encore en soit une manifestation (au sens où il s'agirait essentiellement d'une même âme, comme c'est le cas de la relation entre âme humaine et âme du monde chez Plotin $\left.{ }^{149}\right)$. Mais l'exposé de Calcidius ne fournit aucune indication en ce sens et nous venons de montrer qu'il n'est pas sûr que Numénius admette effectivement l'existence d'une deuxième âme au niveau cosmique. La seule information qu'il nous donne à exploiter est l'affirmation, difficile à élucider, selon laquelle la matière serait auctrix et patrona de « la partie passible de l'âme, dans laquelle se trouve quelque chose de corporel et mortel et de semblable au corps ${ }^{150} \gg$ (patibilis animae partis, in qua est aliquid corpulentum mortaleque et corporis simile). Qu'est-ce à dire ?

\section{3. c. La matière à l'origine du caractère mauvais de l'âme humaine}

Avant de tenter d'expliquer le sens de l'énigmatique formule auctrix et patrona, la définition donnée de l'âme irrationnelle mérite un éclaircissement.

Nous partons ici de l'hypothèse que la notion de partie de l'âme, plutôt que de deuxième âme, tout d'abord, peut constituer de la part de Calcidius une adaptation de ce qu'il lit chez Numénius, lequel semble plutôt considérer l'âme mortelle du Timée comme une âme distincte de l'âme rationnelle ${ }^{151}$. En cela, nous pouvons supposer que ce qui est ici nommé « partie

148. C'est peut-être justement pourquoi le texte latin ne contient pas de verbe esse lorsqu'il exprime l'idée que Platon a évoqué ces deux âmes : Platonemque idem Numenius laudat, quod duas mundi animas autumet, 1.64-65. En revanche, au paragraphe 300, quand Calcidius résume visiblement cette lecture de Platon, il ajoute le verbe esse, sans doute pour expliciter la manière dont il entend l'interprétation de Numénius. Il n'a sans doute pas perçu la subtilité de sa pensée qui consisterait justement à ne pas attribuer à Platon l'idée de l'existence réelle de deux âmes du monde.

149. Sur ce sujet, voir Helleman-Elgersma 1980.

150. Traduction littérale provisoire, voir le développement qui suit et la traduction définitive adoptée dans l'annexe II.

151. Cf. fr. 44, voir le texte cité n. 72 . Il s'agit toutefois ici d'une simple hypothèse de lecture. Nous ne souhaitons pour l'instant rien affirmer sur ce sujet. 
passible de l'âme »ou, pour le rendre plus clairement en français, « partie de l'âme susceptible de passions », correspond à l'âme mortelle du Timée comme siège des passions et, selon toute vraisemblance, à ce que Numénius lui-même considère comme l'âme irrationnelle.

La qualification de ce qui réside en cette âme, ensuite, peut quant à elle être comprise en plusieurs sens. La référence de «ce qu'elle contient de corporel (et donc de mortel) et de semblable au corps » n'est en effet pas claire. Nous pourrions proposer les interprétations suivantes. « Ce qu'elle contient de corporel $\gg$ (aliquid corpulentum) pourrait faire simplement allusion aux sensations dont le corps est à l'origine ou, plus concrètement, en sortant du contexte du Timée, au $\pi v \varepsilon v \tilde{\mu} \alpha$ dont on pourrait imaginer qu'il constitue l'âme passible selon Numénius ${ }^{152}$. L'expression «ce qui est semblable au corps » (corporis simile) pourrait de son côté renvoyer à deux autres idées. Elle rappellerait éventuellement la formule : «partie divisible dans les corps » rencontrée à propos de l'âme du monde, en ce sens que la divisibilité est caractéristique des corps et se retrouverait dans l'âme humaine irrationnelle. Celle-ci aurait alors, comme l'âme cosmique, la matière pour origine selon l'interprétation que nous avons proposé de reconstituer. Toutefois, cette lecture, bien qu'elle ne soit pas impossible, est difficile à maintenir, dans la mesure où, en réalité, le constituant divisible fait partie des ingrédients utilisés par le démiurge pour former l'âme rationnelle ${ }^{153}$. L'expression « ce qui est semblable au corps » renvoie alors peut-être plus simplement aux attitudes de l'âme irrationnelle qui s'adapte aux besoins du corps et finit en cela par lui ressembler, comme le décrit par exemple Socrate dans le Phédon à propos de l'âme en général ${ }^{154}$. Cette dernière interprétation semble la plus convaincante et invite même à relire l'ensemble de l'expression aliquid corpulentum mortaleque et corporis simile. L'adjectif corpulentum, en effet, n'est pas habituel pour exprimer « ce qui est corporel ». On s'attendrait plutôt à lire corporeum. Toutefois, plutôt que de supposer qu'il pourrait ici avoir le sens de «gros, gras» ou de « grand », que l'on retrouve dans le français corpulent, ou encore renvoyer à la notion de condensation, en tant que synonyme de crassior $^{155}$, on peut

152. Voir Bakhouche 2011, p. 854, n. 1060, qui renvoie à Porphyre, Sentence 29.

153. Cela n'empêche toutefois pas Plutarque de faire le rapprochement entre l'âme passionnelle de l'homme et l'âme mauvaise du monde qu'il a associée à ce constituant de l'âme cosmique ; voir, de manière générale, De animae procreatione, \$27-28, 1026 E-1027 A.

154. Phaed. 83d4-6 (chaque plaisir ou chagrin, comme un clou, fixe l'âme au corps et lui

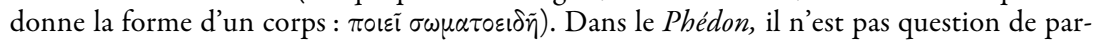
ties de l'âme ni d'une âme irrationnelle proprement dite. Mais les plaisirs et chagrins évoqués sont par excellence les affects de l'âme mortelle du Timée.

155. Voir les différentes acceptions du terme données dans le $T L L$. Le mot peut encore renvoyer à la condensation, comme c'est le cas dans la traduction du Timée proposée par 
remarquer que Calcidius s'en sert précisément dans sa traduction du Timée (p. 31b) pour rendre le grec $\sigma \omega \mu \alpha \tau o \varepsilon i \delta \varepsilon \dot{s}$. Or c'est exactement le mot que Socrate emploie dans le passage du Phédon auquel nous renvoyions ${ }^{156}$. Il est donc possible que l'expression corporis simile soit, de la part de Calcidius, une paraphrase de la formule aliquid corpulentum par laquelle il traduit $\sigma \omega \mu \alpha \tau o \varepsilon i \delta \dot{\varepsilon} s$, auquel cas mortale serait conçu comme un synonyme de corpulentum et le et qui relie les deux formules aurait valeur épexégétique. Nous traduirions alors l'expression par : « la partie de l'âme susceptible de passions dans laquelle se trouve quelque chose de corporel et mortel, c'est-àdire de semblable au corps $\gg$.

Quoi qu'il en soit, c'est cette affinité de l'âme humaine susceptible de passions avec le corps qui contribue à justifier que la matière soit considérée comme son auctrix. Le terme pourrait alors être pris au sens fort de principe constitutif et ici plus précisément de matériau constitutif. Auctrix traduirait alors le grec $\tau \imath \theta \dot{\eta} \nu \eta$ et patrona, qui a pour étymologie pater, revêtirait son sens premier de « mère ». Une telle analyse ne rend toutefois pas pleinement compte de la relation que Numénius envisage entre la matière et l'âme passible, car si l'âme passible est semblable à un corps, elle n'est justement pas un corps, mais a seulement quelque chose en elle qui l'en rapproche. Le sens du terme auctrix doit donc faire l'objet d'une analyse plus approfondie. Nous avons déjà par ailleurs tenté une telle explicitation, une première fois en renvoyant entre autres à la doctrine hébraïque sur laquelle Numénius semble s'appuyer d'après la suite du passage de Calcidius, à la doctrine de l'hérétique platonisant Hermogène telle que Tertullien la présente et à celle de Philon dont même Calcidius se fait l'écho ${ }^{157}$; une

Calcidius lui-même, 49c. Mais il est évident que ce n'est pas le sens à l'œuvre ici. On remarquera que Tertullien emploie aussi corpulentum dans le Contre Hermogène, 19.

156. Phaed. $83 \mathrm{~d} 6$, voir n. 154 . Il ne s'agit évidemment pas de dire qu'il y a ici allusion à ce passage, mais l'idée qui s'y trouve exprimée est sans doute à l'œuvre.

157. Voir Jourdan 2014. Nos conclusions peuvent être brièvement résumées ainsi. Il s'agit de comprendre en quel sens la matière peut être considérée comme auctrix de l'âme irrationnelle, c'est-à-dire de l'âme mortelle considérée comme simple principe de vie des êtres animés. Dans cet article à paraître, nous avons entre autres proposé de comparer cette conception à trois autres, toutes émanant d'une source hébraïque que Numénius a pu connaître. (1) Dans le paragraphe 300 où il résume à sa manière la pensée de Pythagore et de Numénius qu'il vient de rapporter, Calcidius rappelle la parenté de cette pensée avec celle des Hébreux selon lesquels Dieu aurait donné aux homme une âme raisonnable et aux animaux une âme dépourvue de raison, issue de la matière (ex silva rationis expertem). D'après le contexte, il faut comprendre que cette âme dépourvue de raison correspond à l'âme mauvaise en l'homme. Seul nous intéresse ici le sens de l'expression ex silva et l'idée qu'elle semble véhiculer d'une origine «matérielle » de cette âme. L'ensemble du texte latin montre

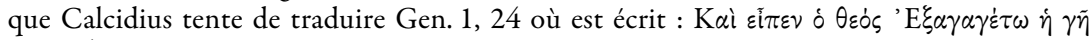

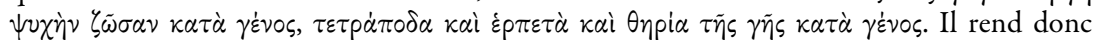
vraisemblablement $\gamma \tilde{\eta}$ par silva et laisse entendre que, sous le commandement de Dieu, la 
terre-matière a produit 《l'âme vivante » ( $\psi v x \grave{\eta} \nu \zeta \tilde{\omega} \sigma \alpha v)$, c'est-à-dire l'âme qui assure les fonctions vitales. Plutôt que d'une affirmation de l'origine matérielle au sens de «corporelle » de l'âme irrationnelle, il s'agit avant tout ici d'une explicitation de la conception biblique à l'œuvre. Calcidius a pu trouver cette citation chez Numénius, non seulement familier de citations bibliques, mais peut-être aussi de leur réécriture en fonction de son propos (voir le fr. $30=$ Porphyre, De antro nympharum, 10). Un autre passage peut confirmer cette origine numénienne de la comparaison et le sens figuré de l'origine matérielle prêtée à l'âme irrationnelle dans l'exposé de Calcidius. L'enseignement hébraïque est encore évoqué au paragraphe 55 où il est question de la vie (vita) que Dieu tire de la voûte céleste (ex convexis caelestibus) pour la donner à l'homme avant d'y insuffler sa propre haleine qui fait naître en lui la raison. Si l'on considère que vita correspond ici à l'âme dépourvue de raison qui toutefois assure les fonctions vitales des êtres animés, on sera ici encore frappé par son origine apparemment « matérielle » (ex convexis caelestibus), par contraste avec l'origine purement divine de la raison (Calcidius interprète ici Gen. 2, 7). Or l'expression peut ici témoigner d'une tentative d'harmonisation du Timée et de la Genèse et en cela remonter à Numénius. Elle sert en effet par ailleurs à Calcidius à traduire les mots $\pi \dot{\alpha} \nu \tau \varepsilon \zeta$ ö $\sigma o l ~ \tau \varepsilon \pi \varepsilon p l-$ $\pi \circ \lambda \circ \tilde{\sigma} \sigma v$ фavep $\tilde{s}$ qui, dans le Timée (41a3), désignent les dieux astraux appelés à devenir les aides du démiurge et plus précisément les fabricants de l'âme mortelle. L'origine « matérielle » de l'âme irrationnelle ne renvoie donc pas à une source corporelle de l'âme rationnelle, mais vraisemblablement à l'œuvre, soumise à la nécessité, de ces dieux démiurges dans l'épisode correspondant du Timée (voir n. 124). Calcidius a pu relever cette conception justement pour distinguer, en l'homme, ce qui provient de Dieu (la raison) de ce qui provient de la nature. Une telle interprétation a pu déjà figurer chez Numénius. (2) Cette idée d'une origine «matérielle » de l'âme, par opposition à l'origine spirituelle et divine de la raison ou de l'esprit (spiritus), dans le cadre toujours d'une exégèse de Gen. 2, 7, se retrouve du moins chez Hermogène (voir Tertullien, De anima, XI, 2; Adversus Marcionem, II, 9), qui semble ne pas hésiter à transformer le texte de la Septante pour

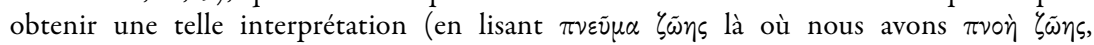
Hermogène réservant le premier terme au souffle divin, le second au souffle naturel. On notera toutefois qu'une telle variante dans le texte biblique apparaît aussi chez Philon lorsqu'il paraphrase le texte, voir Det. 80, Spec. IV, 123, Her. 55-57; $f$. Josèphe, Antiquités juives, I, 34). Ici comme chez Calcidius, la distinction vise essentiellement à désigner ce qui, en l'homme, relève effectivement de Dieu. Elle n'impose pas l'idée d'une origine « corporelle » de l'âme, d'autant moins que la matière, chez Hermogène, n'est pas corporelle (voir n. 27). (3) Une telle distinction peut émaner de Philon dont Numénius semble avoir connu la pensée (voir p. ex. Switalski 1902, p. 42-43; Puech 1934, p. 763-764; Waszink 1966, p. 50-51, n. 4). Philon veut en effet résoudre la contradiction apparente des propos de Gen. 4, 10 et Lev. 17, 11, qui font du sang l'ov̉oi $\alpha$ de l'âme, avec ceux de Gen. 2, 7 où c'est le $\pi \nu \varepsilon \tilde{v} \mu \alpha$ divin qui joue ce rôle (Philon, lui aussi, écrit $\pi v \varepsilon v \tilde{u} \alpha$ là où la Septante, comme il le sait par ailleurs [voir Leg. all. I, 33, 5-7, I, 42] a $\pi v 0 \eta \dot{)}$. Pour cela, il distingue la faculté vitale de la faculté rationnelle, c'est-à-dire l'âme au sens de principe de vie de l'âme au sens de raison ou intellect, dont l'une a pour origine le sang et l'autre le souffle divin (voir Det. 79-84, cf. Spec. IV, 123, Her. 55-57). Calcidius évoque cette distinction au paragraphe 219. Or, là encore, même si Bakhouche 2011, p. 796 n. 562, est d'avis qu'il la connaîtrait par le biais d'Origène, il n'est pas impossible selon nous qu'il la tienne de Numénius (peut-être par l'intermédiaire de Porphyre). - Dans ces trois cas, l'idée d'une origine «matérielle» de l'âme, et plus précisément de l'âme irrationnelle conçue comme principe vital, relève avant tout de l'exégèse biblique et de la tentative d'harmonisation de la pensée hébraïque avec l'enseigne- 
seconde fois en rapprochant la pensée ici exprimée des développements eschatologiques et astrologiques de Macrobe, des Oracles chaldaïques et des textes hermétiques à propos du rapport entre le corps astral, envisagé comme matière d'un certain type, et l'âme irrationnelle qui semble apparaitre lorsque l'âme rationnelle revêt ce corps ou « rencontre » la matière qui le constitue ${ }^{158}$. Nous proposons cette fois d'examiner uniquement ${ }^{159} \mathrm{le}$ sens que peut recouvrir le terme dans le contexte cosmologique de l'interprétation du Timée où il apparaît chez Calcidius et des trois identifications de la matière que Numénius y propose.

Nous venons d'entrevoir, tout en la laissant de côté faute de pouvoir l'étayer par des indications explicites, l'idée que Numénius pouvait penser à la matière productrice de l'âme passible en l'homme au sens où, en tant qu'âme mauvaise du monde, elle fournirait par elle-même cette âme qui en constituerait une partie, en serait le produit ou une manifestation. Nous avons aussi déjà fait allusion à l'idée que la matière, en tant que renvoyant au matériau spatial et nourricier du Timée, pouvait être considérée comme la «nourrice » et la «mère » de cette âme mortelle. Toutefois, pour qu'une telle interprétation soit valable, il faut considérer ce matériau moins comme la matière première que comme le lieu où une telle âme apparaît. L'âme mortelle du Timée n'a en effet pas lieu d'être et n'est justement pas évoquée avant que ne soit introduit le «troisième genre ». L'interprétation proposée est alors possible si l'on rappelle le sens premier du terme auctrix. Il ne signifie pas que la matière serait l'auteur ou le principe de l'âme mortelle - un tel sens n'est que dérivé. Il indique en fait que la matière est ce qui «fait croître » cette âme (augeo) ou en «garantit l'existence», voire en est « responsable ${ }^{160}$. C'est donc essentiellement ce rôle de lui fournir entre autres un lieu où croître qui justifierait que la matière soit considérée comme l'auctrix de l'âme passible au sens où elle est garante de son existence. Il ne s'agit donc pas seulement d'un lieu où être, mais bien

ment grec sur la distinction entre âme rationnelle ou intellect et âme irrationnelle ou principe de vie.

158. Voir Jourdan 2013a-b. Nous avons entre autres comparé là l'enseignement prêté par Jamblique à Numénius dans le fragment 43 sur la matière à l'origine du mal et la théorie du corps astral telle que la présente Macrobe dans son Commentaire du Songe de Scipion, I, 11, 10-12 ; I, 12, 13-15. Macrobe décrit en effet la manière dont, lors de sa descente à travers les sphères planétaires, l'âme rationnelle s'enveloppe d'un corps astral et produit à cette occasion les facultés psychiques destinées à permettre sa vie unie au corps terrestre, ces facultés pouvant, bien qu'elles contiennent le raisonnement, être considérées comme l'âme irrationnelle (nous simplifions). Une telle conception pourrait expliquer le rôle prêté par Numénius à la matière dans le mal qui touche l'âme avant même qu'elle ne s'incarne (voir le fr. 49). Il n'est toutefois pas possible de prouver qu'elle remonte effectivement à lui chez Macrobe ni même qu'il l'ait effectivement développée.

159. Nous développons ici une idée seulement esquissée dans l'article de 2014.

160. Voir Jourdan, 2013a-b, n. 21. 
d'un lieu d'être, c'est-à-dire que la matière est essentiellement la cause de cette âme et que, comme la $\chi \dot{\omega} \rho \alpha$ précisément, elle joue en outre un rôle constitutif. On peut même aller jusqu'à considérer qu'elle provoque d'une certaine façon son existence, au sens où elle la requiert afin que l'âme rationnelle puisse communiquer avec le corps qu'elle lui impose.

L'association de la matière à la nécessité semble toutefois la plus éclairante pour comprendre ce rôle d'auctrix prêtée à la première à l'égard de l'âme passible. Dans le Timée, avons-nous dit, ce sont les dieux astraux qui sont à l'origine de cette âme. Or cette observation ne sert pas seulement à justifier une explication astrologique et eschatologique de l'apparition de l'âme irrationnelle par la notion de corps astral. À l'interprète qui reste dans le cadre étroit du Timée, nous avons vu qu'elle rappelle que ces dieux fabriquent l'âme mortelle en se soumettant complètement à la nécessitéé ${ }^{161}$. En dernière instance, c'est donc bien la nécessité qui est véritablement à l'origine de cette âme, au sens où c'est ultimement elle qui en impose l'existence et fournit les règles selon lesquelles la fabriquer.

En résumé, on pourrait dire que la matière, ainsi identifiée au réceptacle du Timée, à la nécessité, et par suite à la dyade indéterminée, est auctrix de l'âme irrationnelle dans tous les sens que peut revêtir le terme auctrix: en tant que dyade, conçue d'une part comme principe ultime du monde aux côtés du démiurge et assurant d'autre part la fonction d'un principe de séparation et d'apparition du mal, la matière est le principe (auctrix) métaphysique à l'origine à la fois du caractère second de cette âme aux côtés de l'âme rationnelle et de son aspect mauvais, c'est-à-dire ici sans doute du fait qu'elle est soumise aux passions; en tant que nécessité, elle est à l'origine d'un côté de son caractère nécessaire précisément au sein du monde sensible (elle est auctrix au sens où elle impose son existence) et, de l'autre, des mécanismes qui régissent sa formation (elle est auctrix comme responsable de cette constitution); en tant que réceptacle du devenir enfin, elle lui fournit à la fois un lieu d'apparition qui garantit son existence et un matériau protecteur et nourricier, les dieux astraux se servant des différentes parties du corps pour assurer le bon fonctionnement de ses différents mécanismes (elle est auctrix comme garante) ${ }^{162}$. L'identification de la matière à l'âme mauvaise du monde peut quant à elle être considérée comme servant utilement la pensée analogique puisque, sans poser réellement l'existence d'une deuxième âme du monde, elle suggère néanmoins qu'une « âme », la ma-

161. Voir n. 124. Ce n'est que pour son emplacement qu'ils ne sont plus complètement soumis à la nécessité, mais là, à l'imitation de leur « père », ils font œuvre de réflexion en vue du meilleur et ainsi séparent l'âme mortelle de l'âme rationnelle située dans la tête (voir

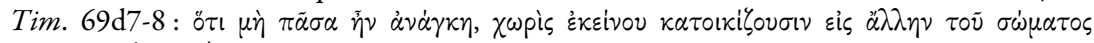

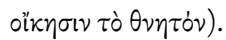

162. Le système du cœur, par exemple, sert à protéger la partie agressive (Tim. 70b-d). 
tière $(s i c)$, est productrice ou garante d'une autre âme - idée que Numénius a pu également soutenir, mais qui n'est exprimée dans aucun des témoignages qui nous sont parvenus.

En ce qui concerne le substantif patrona, il vient sans doute nuancer le terme auctrix. Il semble servir à préciser que ce dernier ne signifie pas que la matière serait l' « auteur » de l'âme passible, au sens où elle aurait créé celleci, mais indique seulement qu'elle est la garante de son existence. Le terme patrona signifie quant à lui que la matière serait la « protectrice » et l'«appui » de l'âme passible. Ce mot typique du vocabulaire latin peut constituer un ajout de Calcidius à son modèle pour expliciter le sens d'auctrix traduisant quant à lui une notion grecque difficile à comprendre ${ }^{163}$. Toutefois comme, au masculin, il sert à l'origine à désigner le maître d'un affranchi ou le protecteur des plébéiens, le terme suggère habilement que l'âme passible est, sinon complètement serve, du moins fortement dépendante de la matière. Cela suffit à permettre de conclure, sinon à son vice essentiel, puisque Numénius ne parle pas ici explicitement d'âme humaine mauvaise, du moins à sa tendance essentiellement mauvaise. On pourrait penser en effet que, de même que Numénius rappelle que la nécessité finit par se soumettre à la raison démiurgique, il n'est pas impossible qu'il ait envisagé que cette âme aussi se soumette à l'âme rationnelle, tout comme, dans le Timée, une fois le moment chaotique de la naissance passé, l'ordre finit par s'installer dans l'âme.

Dans le cadre de la troisième identification qui conduit à assimiler la matière à l'âme mauvaise du monde et à envisager par suite la matière comme garante de l'existence de l'âme irrationnelle en l'homme, nous parvenons à la conclusion suivante. C'est selon nous par le caractère originel de cette relation de soumission de l'âme irrationnelle à la matière, dont la malfaisance a été fortement soulignée, que Numénius explique le mal qui, en l'homme, finit par atteindre l'âme rationnelle de l'extérieur (l'âme irrationnelle étant en lien direct avec elle pour lui transmettre les sensations du corps) et justifie donc une troisième fois le rôle de la matière dans cette affection. Quant à ce mal proprement dit, il se manifeste d'une part dans l'apparition de cette seconde âme qui, au moment de l'incarnation, vient s'ajouter à l'âme rationnelle, et d'autre part dans la dépendance de l'âme irrationnelle envers la matière-nécessité qui finit par entacher même l'âme rationnelle, étant donné sa relation à l'âme irrationnelle qui lui permet de communiquer avec le corps.

163. Il est difficile de savoir quel mot grec Calcidius a voulu traduire. Dans son index ad commentarium, Waszink 1962 ne répertorie pas auctrix. Nous pourrions penser à $\tau \imath \theta \dot{\eta} \nu \eta$, mais Calcidius utilise visiblement nutrix pour rendre ce mot. 


\section{Conclusion}

Dans le fragment 52, la matière est tour à tour assimilée à la dyade indéterminée, à la nécessité et à l'âme mauvaise. Ces trois identifications ou analogies explicatives permettent d'envisager sur le plan métaphysique et cosmologique le rôle que Numénius lui attribue dans l'origine du mal (tant au niveau cosmique qu'humain) et plus précisément dans l'incarnation de l'âme humaine, tel que Jamblique le rapporte à la fin du fragment 43.

Ces identifications sont tout d'abord permises par le sens que le terme ư $\lambda \eta$ revêt depuis Aristote. Sans perdre sa référence au matériau constitutif qu'est le réceptacle du Timée, ce mot en vient à désigner principalement une entité distincte d'une part de la forme et de la finalité, qui constituent l'essence véritable de tout objet ou phénomène, et d'autre part de l'agent, qui permet à ce phénomène de s'actualiser. Dans ce cadre, la ü $\lambda \eta$ représente le substrat du changement, la cause matérielle et donc les conditions de possibilité de tout phénomène. Cette définition permet de l'assimiler au deuxième principe de la pensée pythagoricienne et platonicienne, qui est alors nommé « dyade » lorsqu'il est envisagé au niveau métaphysique et désigné en termes conformes à l'enseignement oral attribué à Platon, et représenté par la nécessité quand il est envisagé au niveau cosmique et conçu comme correspondant aux causes de type inférieur décrites dans le Timée. Ces identifications sont en outre régies d'un côté par le désir de rendre compte de l'enseignement de l'ancien pythagorisme et de l'ancienne Académie d'où émanent respectivement l'identification du deuxième principe à la source du mal et la référence à la dyade indéterminée; et, d'un autre côté, par le souci de rester fidèle à Platon en interprétant ses textes les uns par les autres - d'où l'association ultime de la matière ainsi conçue à l'âme mauvaise pour donner une cause positive au mouvement désordonné qu'elle produit.

Ainsi, en tant que dyade indéterminée, d'abord, la matière est une source du mal extérieure à l'âme rationnelle, au niveau cosmique parce qu'elle s'oppose à la rationalité incarnée par le démiurge et au niveau humain parce qu'en tant que principe de séparation, de division et de multiplication, elle sépare l'âme rationnelle du divin (en lui imposant l'incarnation) et lui accole une deuxième âme. En tant que nécessité, elle joue ce même rôle au niveau cosmique comme source du mouvement désordonné résiduel dans le monde engendré et au niveau humain en ce qu'elle est à l'origine même de l'incarnation de l'âme rationnelle et de la formation de l'âme irrationnelle dont elle s'avère aussi la cause des passions désordonnées. En tant qu'âme mauvaise, elle est enfin la cause positive du désordre cosmique. Devenant, à ce stade de l'analogie, responsable aussi de l'âme irrationnelle en l'homme (qu'elle lui fournisse son matériau premier ou que, en tant que réceptacle du devenir, dyade et nécessité, elle en garantisse 
simplement l'existence parce qu'elle lui donne un lieu d'apparition, provoque cette existence et en régit les mécanismes), elle crée une relation de dépendance essentielle de cette âme irrationnelle à son égard, qui explique que celle-ci puisse être conçue comme le mal qui affecte effectivement l'âme rationnelle et dont l'origine reste pourtant la matière.

Toutes ces identifications explicitant la relation que l'âme irrationnelle entretient avec la matière au niveau même de sa constitution, c'est-à-dire avant sa manifestation dans le monde sensible, elles contribuent aussi à expliquer comment Numénius a pu concevoir que le mal infecte l'âme rationnelle avant même son incarnation. Rappelons en effet que cette âme doit d'une part nécessairement s'incarner, tant au niveau cosmique qu'humain, en vertu respectivement de sa fonction et des mécanismes du monde sensible pour lequel elle est formée. Elle se voit d'autre part, au niveau humain, nécessairement ajouter l'âme irrationnelle pour lui servir d'intermédiaire dans la relation au corps imparfait de l'homme. En cela, Jamblique semble avoir raison de considérer que la matière, chez Numénius, est non seulement cause du mal pour l'âme rationnelle, mais aussi de son incarnation, que Numénius aurait selon lui envisagée comme nuisible dans tous les cas.

Une telle reconstitution de la doctrine de Numénius pourra sembler audacieuse. Il peut en effet paraître inadéquat de vouloir rétablir la cohérence d'une pensée parvenue de manière fragmentaire et qui se présente elle-même sous une forme multiple. Nous espérons toutefois avoir montré que cela n'est pas impossible, si les bribes parvenues de cet enseignement sont replacées dans leurs contextes respectifs. Nous avons du moins pu examiner ici le sens du propos prêté à Numénius sur la matière au niveau métaphysique et cosmologique. Or, à ce niveau précisément, Numénius s'avère essentiellement héritier de la philosophie platonicienne dont il emprunte le cadre de pensée, la méthode, mais aussi les querelles, comme c'est le cas avec les stoïciens. Et cet héritage, il ne le trahit pas, même lorsqu'il intègre, à titre de confirmation ${ }^{164}$, les leçons des courants orientaux ${ }^{165}$.

164. Sur ce point, voir l'interprétation du fr. 1 par Plese 2005, p. 360 ; Jourdan 2015. Voir aussi Edwards 2010, p. 116.

165. Voir la note 154 et Jourdan 2013a-b, c, 2014, 2015 sur les autres interprétations du terme auctrix. On remarquera à cette occasion la proximité avec Plutarque. Même si les deux philosophes parviennent à des conclusions distinctes relativement à l'origine du mal, on retrouve chez eux la même méthode exégétique dans l'interprétation de Platon et la même opposition aux stoïciens, voire à certains pythagoriciens. Plutarque recourt en outre lui aussi aux enseignements orientaux, comme dans le De Iside et Osiride, où il évoque le dualisme iranien. Mais il le transforme pour le faire correspondre à sa manière d'interpréter Platon, découvrant ainsi étonnamment en lui la présence d'un troisième principe (sous la forme de Mithra, censé être le médiateur entre Ariman et Horomazès, $₫ 46,396$ D-E). Numénius en reste de son côté à une forme de dualisme, mais l'ancre toujours dans sa lecture de Pythagore et de Platon. 


\section{ANNEXES}

I. Extrait du traité de Jamblique Sur l'âme, cité par Stobée, I 49, 37, 76-98, vol. I, p. 374, 21-375 Wachsmuth $=$ Jamblique, De anima, $\$ 23$, p. 48, 11-29 Finamore-Dillon) = Numénius, fr. 43 Des Places

Texte corrigé par rapport à l'édition de É. des Places et complété, la partie centrale étant omise dans cette dernière édition.

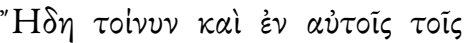

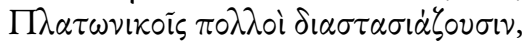

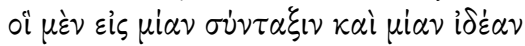

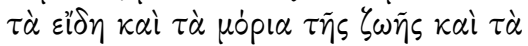

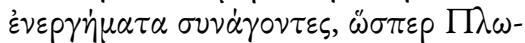

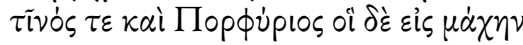

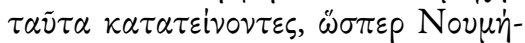

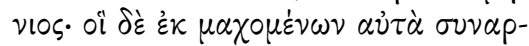

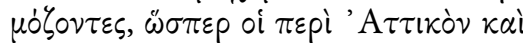

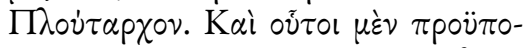

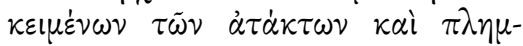

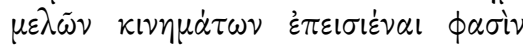

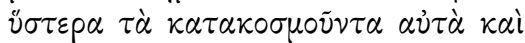

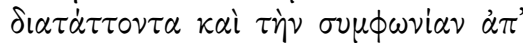

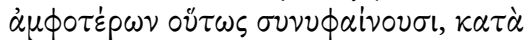

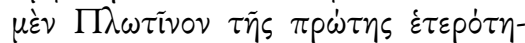

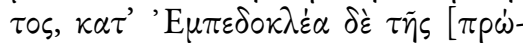

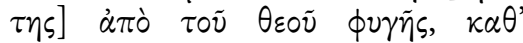

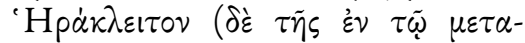
$\beta \dot{\alpha} \lambda \lambda \varepsilon \sigma \theta \alpha \iota \dot{\alpha} \nu \alpha \pi \alpha \dot{\nu} \lambda \eta s, k \alpha \tau \dot{\alpha} \delta \dot{\varepsilon} \tau o \dot{v} s$

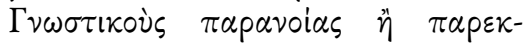

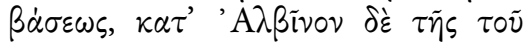

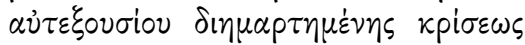

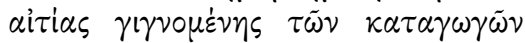

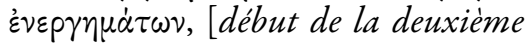
partie du fr. 43 Des Places] $\tau \tilde{\omega} \nu \delta^{\prime} \alpha v^{5}$

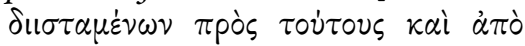
$\tau \tilde{\omega} \nu$ हैं $\xi \omega \theta \varepsilon \nu \pi \rho \circ \sigma \phi \nu \circ \mu \dot{\varepsilon} \nu \omega \nu \pi \rho \circ \sigma \tau \theta \dot{\varepsilon} \nu$ -

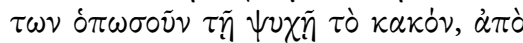

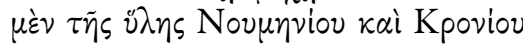

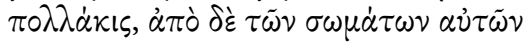

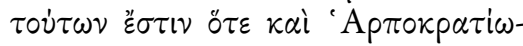

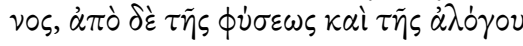

Or déjà, parmi les platoniciens euxmêmes, beaucoup divergent, les uns, comme Plotin et Porphyre, ramenant à un seul système et à une seule forme les espèces et parties de la vie ainsi que ses activités ; les autres, comme Numénius, les opposant radicalement dans un combat; d'autres encore, comme Atticus, Plutarque et leurs pairs, les faisant s'harmoniser à l'issue du combat. Et ceux-ci (scil. Plutarque et Atticus) disent (par ailleurs) que, tandis que les mouvements irréguliers et désordonnés sont déjà là avant, les activités de l'âme qui les ordonnent et les mettent en ordre viennent ensuite s'introduire en eux, et c'est ainsi à partir des uns et des autres qu'ils (scil. les deux philosophes) tissent l'accord, la cause des activités provoquant la descente de l'âme étant, selon Plotin, la première altérité, selon Empédocle, la fuite loin de dieu, selon Héraclite le repos qui consiste dans le changement, selon les gnostiques un écart vis-à-vis de la raison ou du droit chemin, selon Albinus un jugement erroné du libre arbitre ; tandis que d'autres, en désaccord avec les précédents, ajoutent le mal en quelque sorte à l'âme en lui attribuant comme cause ce qui s'attache naturellement à elle de l'extérieur, ainsi, Numénius et Cronius le font souvent venir de la matière, ces derniers parfois, ainsi qu'Harpocration, des corps eux-mêmes ${ }^{166}$, et Plotin et

166. Sur cette traduction qui differe des traductions traditionnelles du passage, voir Jourdan 2013a-b. 


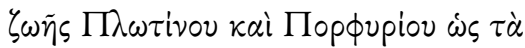
$\pi \circ \lambda \lambda \dot{\alpha}$.

Porphyre, le plus souvent, de la nature et de la vie irrationnelle.

II. Calcidius, Commentaire du Timée, 299-300 Waszink

Les paragraphes 295-299 correspondent au fr. 52 de Numénius dans l'édition de É. des Places. La numérotation des lignes de cette édition est ici intégrée au texte.

295. Nunc iam Pythagoricum dogma recenseatur. [2] Numenius ex Pythagorae magisterio Stoicorum hoc de initiis dogma refellens Pythagorae dogmate, cui concinere dicit dogma Platonicum, [5] ait Pythagoran deum quidem singularitatis [nomine] nominasse, siluam uero duitatis. Quam duitatem indeterminatam quidem minime genitam, limitatam uero generatam esse dicere: hoc est, antequam exornaretur quidem formamque et ordinem [10] nancisceretur, sine ortu et generatione, exornatam uero atque inlustratam a digestore deo esse generatam. atque ita, quia generationis sit fortuna posterior, inornatum illud minime

$295^{167}$. Passons donc maintenant en revue la doctrine pythagoricienne. Numénius, qui vient de l'école de Pythagore, réfute la doctrine stoïcienne des principes dont nous venons de parler en recourant à celle de Pythagore avec laquelle, selon lui, s'accorde la doctrine de Platon. Il dit que Pythagore a donné au dieu le nom de «monade », à la matière, celui de « dyade ». Indéterminée, cette dyade, aurait-il affirmé $^{168}$, n'est pas engendrée, mais limitée, elle est engendrée. Cela signifie qu'avant d'être parée et de recevoir forme et ordre, elle serait sans naissance ni génération, tandis qu'une fois parée et embellie par le dieu démiurge, elle serait engendrée. Et ainsi, comme ce qui advient par la génération est postérieur $^{169}$, il faudrait comprendre que

167. Les notes qui accompagnent cette traduction visent essentiellement l'explication de nos choix de traduction et l'explicitation de passages non commentés par ailleurs.

168. Dans tout le texte, les infinitifs latins signalent que Calcidius est en train de rapporter le discours de Numénius. Nous les rendons en français, à titre ici d'expérimentation, par le conditionnel qui permet d'exprimer ce discours indirect. Quand Calcidius abandonne les infinitifs de discours indirect, en revanche, nous revenons à l'indicatif, ces passages pouvant précisément constituer des interprétations ou affirmations de Calcidius lui-même. C'est peut-être par exemple le cas dans les deux premières lignes du paragraphe 297 où il pourrait résumer ce qu'il vient de retranscrire. La suite paraît en revanche toujours empruntée à Numénius, évoqué quelques lignes plus bas (1.60). Calcidius serait resté à l'indicatif, sans doute entraîné dans son élan. Nous marquons ici cette distinction dans la mesure où, concernant l'ensemble des témoignages relatifs à la pensée de Numénius, ces changements de mode sont parfois significatifs : à l'occasion, en effet, ils permettent de distinguer le propos prêté à Numénius de l'interprétation ou du résumé qui en sont faits, ou parfois de repérer des ajouts, comme dans le cas du fragment 31 (voir Jourdan, 2013a, n. 78).

169. Le terme fortuna indique moins l'événement de la génération que la «situation, l'état, le sort » de ce qui est produit par cette génération, c'est pourquoi nous rendons le terme par le verbe advenir. 
generatum aequaeuum deo, a quo est ordinatum, intellegi debeat. [15] Sed non nullos Pythagoreos uim sententiae non recte adsecutos putasse dici etiam illam indeterminatam et inmensam duitatem ab unica singularitate institutam, recedente a natura sua singularitate et in duitatis habitum migrante: non recte, ut quae [20] erat singularitas esse desineret, quae non erat duitas subsisteret, atque ex deo silua et ex singularitate inmensa et indeterminata duitas conuerteretur. quae opinio ne mediocriter quidem institutis hominibus conpetit. Denique Stoicos definitam et limitatam [25] siluam esse natura propria, Pythagoran uero infinitam et sine limite dicere. cumque illi [30] quod natura sit inmensum non posse ad ordinem atque modum redigi censeant, Pythagoran solius hanc dei fore uirtutem ac potentiam adserere, ut quod natura efficere nequeat, deus facile possit, ut qui sit omni uirtute potentior atque praestantior, et a quo natura ipsa uires mutuetur.

296. Igitur Pythagoras quoque, inquit Numenius, fluidam et sine qua- cette dyade ${ }^{170}$ qui n'est ni parée ni engendrée est aussi ancienne que le dieu par qui elle est ordonnée. Mais certains pythagoriciens n'auraient pas correctement saisi le sens de cette idée. Ils auraient cru qu'elle signifiait aussi que cette dyade indéterminée et dépourvue de mesure était produite par la monade, qui était seule à exister ${ }^{171}$, lorsqu'elle quittait sa nature et passait à l'état de dyade. Mais ce n'est pas correct, parce que ce qui existait, la monade, cesserait d'être, tandis que ce qui n'existait pas, la dyade, viendrait à l'être, et du dieu, par une telle transformation, viendrait la matière, de la monade la dyade indéterminée et dépourvue de mesure. Or une telle opinion ne se rencontre même pas chez des hommes médiocrement instruits. Enfin les stoïciens affirmeraient que la matière est définie et limitée par sa nature propre, tandis que Pythagore la dirait infinie et sans limite. Et alors que les premiers considéreraient que ce qui est par nature sans mesure ne peut être amené à un ordre et à une mesure, Pythagore soutiendrait que le dieu est seul à avoir ce pouvoir et cette puissance: il peut aisément accomplir ce dont la nature n'est pas capable parce que c'est lui qui est plus puissant et plus efficace que tout pouvoir et c'est à lui que la nature elle-même emprunte ses forces.

296. Par suite, Pythagore aussi, dit Numénius, est d'avis que la matière est

170. Illud ne peut être rendu par substance (van Winden, Bakhouche, Moreschini) ou être, ni encore par ensemble (Des Places), à moins d'introduire une information supplémentaire dans le texte qui risque d'induire en erreur sur la nature de la dyade en question.

171. L'expression ab unica singularitate ne signifie pas que la monade était unique au sens où l'auteur préciserait ici qu'il n'y avait pas plusieurs monades. Elle signifie que, d'après la pensée prêtée aux pythagoriciens en question, la monade était seule à exister, comme si elle était une « fille unique » (selon l'emploi courant de l'adjectif) et qu'il n'y avait pas de dyade à ses côtés, la dyade résultant ensuite d'une transformation de la monade. 
litate siluam esse censet, [35] nec tamen, ut Stoici, naturae mediae interque bonorum malorumque uiciniam, quod genus illi adpellant indifferens, sed plane noxiam. Deum quippe esse - ut etiam Platoni uidetur - initium et causam bonorum, siluam malorum. At uero quod [40] ex specie siluaque sit, indifferens: non ergo siluam, sed mundum ex speciei bonitate siluaeque malitia temperatum. Denique ex prouidentia et necessitate progenitum ueterum theologorum scitis haberi.

297. Siluam igitur informem et carentem [45] qualitate tam Stoici quam Pythagoras consentiunt, sed Pythagoras malignam quoque, Stoici nec bonam nec malam. Dehinc, tamquam in progressu uiae malum aliquod obuium, perrogati: unde igitur mala? peruersitatem seminarium malorum fore causati sunt. [50] nec expediunt adhuc, unde ipsa peruersitas, cum iuxta ipsos duo sint initia rerum, deus et silua: deus summum et praecellens bonum, silua, ut censent, nec bonum nec malum. Sed Pythagoras adsistere ueritati miris licet et contra opinionem hominum [55] operantibus adseuerationibus non ueretur: qui ait existente prouidentia mala quo- fluide et sans qualité, toutefois il ne pense pas, comme les stoïciens, qu'elle est d'une nature intermédiaire et constitue la zone voisine entre les biens et les maux ${ }^{172}$, représentant ce genre qu'ils appellent «indifférent », mais elle est selon lui entièrement nuisible. De fait, le dieu, pour lui, comme aussi pour Platon, serait principe et cause des biens, la matière des maux. Et c'est en revanche ce qui vient de la forme et de la matière qui serait indifférent : non pas la matière, donc, mais le monde, qui est composé de la bonté de la forme et de la malfaisance de la matière. C'est pourquoi il passerait pour né de la providence et de la nécessité dans les enseignements des anciens théologiens.

297. Sur le fait que la matière, donc, soit privée de forme et de qualité, les stoïciens et Pythagore sont d'accord, mais Pythagore ajoute qu'elle est malfaisante, tandis que pour les stoïciens, elle n'est ni bonne ni mauvaise. De là, quand, en cours de route, pour ainsi dire, ils rencontrent quelque mal et qu'on leur demande : « d'où viennent donc les maux ? », ils allèguent qu'une perversion sera à l'origine des maux. Mais ils n'expliquent pas encore d'où vient cette perversion elle-même, puisque, selon eux, les principes des choses sont au nombre de deux, le dieu et la matière, le dieu étant le bien suprême et excellent, la matière, à leur avis, n'étant ni bien ni mal. Mais Pythagore ne craint pas d'assister la vérité ${ }^{173}$ même par des affirmations étonnantes et qui vont à l'encontre de l'opinion com-

172. Le terme viciniam étonne parce qu'il s'agit d'un substantif, là où l'on aurait attendu l'adjectif vicinam accordé avec silvam. Aucun manuscrit ne comporte de variante et personne n'a jusqu'ici proposé de correction. Il ne semble pourtant pas impossible que le second $i$ ait été ajouté par inadvertance. Si cette correction était acceptée, nous traduirions : « [...] et que, située entre les biens et les maux, elle est voisine des uns et des autres [...] ».

173. L'image est celle de l'avocat qui assiste son client au tribunal. 
que necessario substitisse, propterea quod silua sit et eadem sit malitia praedita. Quod si mundus ex silua, certe factus est de existente olim natura maligna. proptereaque [60] Numenius laudat Heraclitum reprebendentem Homerum, qui optauerit interitum ac uastitatem malis uitae, quod non intellegeret mundum sibi deleri placere, siquidem silua, quae malorum fons est, exterminaretur. Platonemque idem Numenius [65] laudat, quod duas mundi animas mune: selon lui, en effet, bien que la providence existe, il est nécessaire que les maux aussi subsistent ${ }^{174}$ parce qu'il y a une matière et que cette matière est dotée de malfaisance. Or si le monde provient de la matière, il a assurément été fait à partir de la nature malfaisante qui existait autrefois ${ }^{175}$. Voilà aussi pourquoi Numénius loue Héraclite lorsqu'il reproche à Homère d'avoir souhaité aux maux qui affectent la vie anéantissement et ruine: il ne comprenait pas que c'était approuver la destruction du monde puisqu'en effet

174. L'expression mala necessario substitisse renvoie au Théétète, 176a5-6 : 'A $\mathrm{A} \lambda$ ' oй $\tau$ '

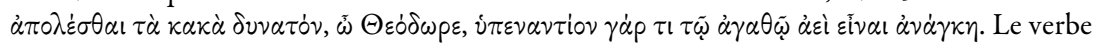
substitisse doit être rendu très exactement car il est essentiel dans l'histoire de l'interprétation du passage. Il traduit en effet le terme í $\pi \varepsilon v \alpha v \tau i o v$ dont certains interprètes de Platon se servent pour définir la nature du mal dont Socrate affirme ici la nécessité de l'existence. Proclus (De malorum subsistantia, 52-54), par exemple, l'utilise pour expliquer que le mal n'est pas à proprement parler « contraire » au bien, mais « subcontraire », c'est-à-dire qu'il correspond selon lui à une privation qui coexiste avec le bien et, par cette coexistence, partage la forme et le pouvoir de ce bien dont il tire précisément sa force. Par cette notion de « subcontraire », Proclus peut maintenir l'idée de la présence d'une contrariété entre les maux particuliers et le bien, sans attribuer un être indépendant au mal (voir Isaac 1982, p. 31). Concernant Numénius, l'expression montre que, dans sa réflexion sur le mal, il fait usage des passages de Platon classiquement cités dans les écoles pour la traiter. La même remarque est valable à propos de l'expression existente olim natura maligna (1.59) qui renvoie quant à elle au passage du mythe du Politique : voir la note suivante.

175. L'expression renvoie certes à la dyade qui est aussi ancienne que le dieu (1. 14-15), mais surtout à l'«antique nature »du mythe du Politique. Dans ce texte, l'étranger qui narre le mythe explique que la perte, par le monde, du mouvement bien orienté que lui avait

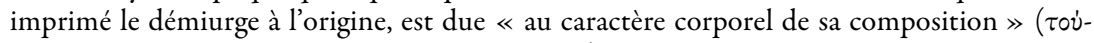

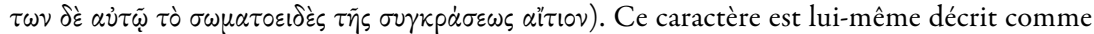
associé à son « antique nature qui participait d'un grand désordre avant d'avoir été conduite

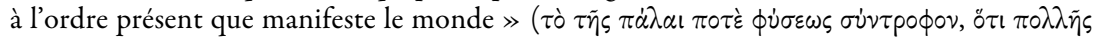

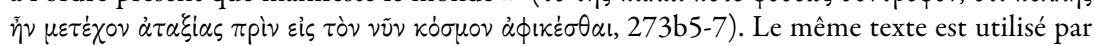
Plutarque qui, quant à lui, identifie l'antique nature non pas à la matière, comme le fait $\mathrm{Nu}$ ménius, mais à l'aspect mauvais que l'âme du monde aurait eu selon lui à l'origine. Ainsi, en se référant à l'enseignement non écrit sur la dyade, au Timée sur la matière, la nécessité et le chaos précosmique, aux Lois sur l'hypothèse d'une deuxième âme du monde qui serait mauvaise (voir la note suivante), au Théétète (voir la note précédente), au Politique, mais aussi au Philèbe où apparaît la notion d'illimité conçu comme l'un des quatre grands genres $\mathrm{du}$ monde à côté de la limite $(23 \mathrm{c} 9)$, Numénius recourt visiblement à un dossier de textes utilisés couramment dans les écoles platoniciennes pour traiter de la question du mal chez Platon. On retrouve les mêmes référence chez Plutarque, De animae procreatione, 6, 1014 D$1015 \mathrm{C}$ qui les utilise pour soutenir la thèse inverse à cette interprétation pythagorisante donnée par Numénius. 
autumet, unam beneficientissimam, malignam alteram, scilicet siluam. Quae licet incondite fluctuet, tamen, quia intimo proprioque motu mouetur, uiuat et anima conuigetetur necesse est lege eorum omnium, quae [70] genuino motu mouentur. Quae quidem etiam patibilis animae partis, in qua est aliquid corpulentum mortaleque et corporis simile, auctrix est et patrona, sicut rationabilis animae pars auctore utitur ratione ac deo. Porro ex deo et silua factus [75] est iste mundus.

298. Igitur iuxta Platonem mundo bona sua dei tamquam patris liberalitate conlata sunt, mala uero matris la matière, qui est la source des maux, serait par là exterminée. Le même Numénius loue aussi Platon pour avoir clairement parlé de deux âmes du monde ${ }^{176}$, l'une absolument bienfaisante, l'autre malfaisante, à savoir évidemment la matière ${ }^{177}$. Car celle-ci, malgré son agitation désordonnée, cependant, parce qu'elle se meut d'un mouvement intérieur qui lui est propre $^{178}$, doit nécessairement vivre et recevoir la vie d'une âme d'après la loi qui régit tout ce qui se meut d'un mouvement inné. Celle-ci est en outre aussi la garante et protectrice ${ }^{179}$ de la partie de l'âme susceptible de passions dans laquelle se trouve quelque chose de corporel $^{180}$ et mortel, c'est-à-dire de semblable au corps, de même que la partie rationnelle de l'âme a pour garants la raison et le dieu. Au surplus, c'est du dieu et de la matière ${ }^{181}$ qu'est fait ce monde.

298. D'après Platon, donc ${ }^{182}$, ses biens ont été prodigués au monde par la générosité du dieu comme s'il était un père, mais les maux se sont attachés à

176. Leg. X, 896e4-6, 897d1.

177. Tel qu'il est transmis, le passage montre clairement que Numénius n'accorde pas l'existence de deux âmes du monde à proprement parler, puisqu'il identifie la seconde à la matière. En cela, il reste en accord avec le texte de Platon. Voir les remarques sur ce point dans le corps de l'article.

178. C'est-à-dire qu'elle se meut par elle-même et doit donc être pourvue d'une âme.

179. Sur cette traduction qui tend à revenir au sens originel de ces deux termes, voir les remarques dans le corps de l'article.

180. Sur ce terme, voir aussi les remarques dans le corps de l'article.

181. On sera sensible à l'emploi de ex dans tout ce texte. Calcidius n'emploie pas la préposition a pour distinguer l'agent divin du matériau dyade. La préposition a n'apparât qu'au paragraphe 300, dans la transcription de l'enseignement hébraïque où elle permet justement de distinguer Dieu comme agent du matériau dont est fait l'âme humaine, introduit par ex. Le fait d'employer la même préposition pour désigner la double origine du monde tend au contraire, dans l'exposé emprunté à Numénius, à souligner que le dieu et la matière ont également valeur de principes, même si, in fine, le dieu s'avère supérieur et soumet la matière à l'ordre.

182. Rappelons que Platon est censé avoir suivi Pythagore (1.4). Ces rappels montrent bien que Numénius tend avant tout à interpréter Platon et que le recours à Pythagore est un moyen dans cette entreprise : voir n. 9. 
siluae uitio cohaeserunt. Qua ratione intellegi datur Stoicos frustra causari nescio quam [80] peruersitatem, cum quae proueniunt ex motu stellarum prouenire dicantur. stellae porro corpora sunt ignesque caelites. omnium quippe corporum silua nutrix est, ut etiam quae sidereus motus minus utiliter et inprospere turbat originem trabere uideantur [85] ex silua, in qua est multa intemperies et inprouidus inpetus et casus atque ut libet exagitata praesumptio. Itaque si deus eam correxit, ut in Timaeo loquitur Plato, redegitque in ordinem ex incondita et turbulenta iactatione, certe confusa [90] haec intemperies eius casu quodam et inprospera sorte habebatur nec ex prouidentiae consultis salubribus. Ergo iuxta Pythagoran siluae anima neque sine ulla est substantia, ut plerique arbitrantur, et aduersatur prouidentiae consulta eius iupugnare [95] gestiens malitiae suae uiribus. Sed prouidentia quidem est dei opus et officium, caeca uero fortuitaque temeritas ex prosapia siluae, ut sit euidens iuxta lui par le vice de la matière, sa mère. Aussi est-il donné de comprendre qu'il est vain, de la part des stoïciens, d'alléguer je ne sais quelle perversion quand ils disent que ce qui arrive, arrive à cause du mouvement des étoiles. Les étoiles, d'ailleurs, sont des corps et feux célestes. Or, de tous les corps, la matière est la nourrice, si bien que les troubles, sources de nuisances et de malheurs, provoqués par le mouvement des étoiles, tirent manifestement leur origine de la matière, où se trouve une grande instabilité ${ }^{183}$, un élan inconsidéré, du hasard, ainsi qu'une présomption qui s'agite comme elle veut. C'est pourquoi, si le dieu l'a redressée, comme dit Platon dans le Timée, et, «d'une agitation désordonnée et turbulente, l'a ramenée à l'ordre » ${ }^{184}$, cette instabilité confuse qui est la sienne était assurément le fruit du hasard et d'un sort malheureux et non des desseins salutaires de la providence. Ainsi donc, selon Pythagore, l'âme de la matière ${ }^{185}$, d'une part, n'est pas sans substance ${ }^{186}$, comme le croit le plus grand nombre, et, d'autre part, elle s'oppose à la providence, brûlant du désir d'attaquer ses desseins par les forces de sa malfaisance. Mais la providence, assurément, est l'œuvre et la fonction du dieu, tandis

183. Le terme intemperies peut aussi avoir une connotation morale. Voir n. 146.

184. Citation de Tim. 30a4-5. L'ordre des mots differre de celui proposé par Calcidius dans sa propre traduction du passage. Calcidius suit sans doute ici le texte de Numénius.

185. En suivant l'identification proposée à la ligne 66-67, on pourrait ici être tenté de traduire « l'âme qu'est la matière » et comprendre que Numénius affirme, contre Aristote peut-être, que cette âme a bien une substance qui est précisément la matière. On remarquera en outre que, dans certains manuscrits, on trouve la leçon silva animae, « la matière de l'âme » : voir n. 147.

186. Depuis Waszink 1962 (p. 300, second apparat), ce passage est habituellement interprété comme exprimant une opposition à Aristote pour qui l'âme est puissance $(D e$ anima, II, 1, 412a9). Pourtant, le terme substantia traduit simplement ov̉oi $\alpha$ et n'implique pas la différence entre puissance et entéléchie comme c'est le cas dans le texte d'Aristote proposé à la comparaison. On peut alors éventuellement comprendre que, selon Numénius, la substance ou le substrat de l'«âme de la matière » est la matière elle-même qu'il vient de décrire (voir la note précédente). D’après Baltes 1975, p. 251, enfin, la phrase renverrait plutôt à un débat au sein de l'école sur la question de savoir si cette deuxième âme existe ou non. 
Pythagoran dei siluaeque, item prouidentiae fortunaeque coetu cunctae rei molem esse constructam. [100] Sed postquam siluae ornatus accesserit, ipsam quidem matrem esse factam corporeorum et natiuorum deorum, fortunam uero eius prosperam esse ex magna parte, non tamen usque quaque, quoniam naturale uitium limari omnino nequiret.

299. [105] Deus itaque siluam magnifica uirtute comebat uitiaque eius omnifariam corrigebat non interficiens, ne natura siluestris funditus interiret: nec uero permittens porrigi dilatarique passim, sed ut manente natura, quae ex incommodo habitu [110] ad prosperitatem deuocari commutarique possit, ordinem inordinatae confusioni, modum inmoderationi et cultum foeditati coniungens totum statum eius inlustrando que l'irréflexion ${ }^{187}$ aveugle et fortuite provient de la souche de la matière. Il en résulte évidemment, selon Pythagore, que la masse de l'univers a été formée par la rencontre du dieu et de la matière comme de la providence et du hasard $^{188}$. Mais après que la parure est venue s'ajouter à la matière, celle-ci serait ${ }^{189}$ elle-même devenue la mère de ce qui est corporel et des dieux engendrés ${ }^{190}$; sa condition serait heureuse en grande partie, mais toutefois pas totalement, puisque son vice naturel n'aurait pu être complètement éliminé.

299. C'est pourquoi le dieu ornait la matière par sa puissance magnifique et, de tous les côtés, en corrigeait les défauts, mais sans les détruire, de peur que la nature matérielle ne pérît complètement, et sans non plus lui permettre de s'étendre et de se dilater partout. Mais, dans l'idée que sa nature devait subsister, comme elle était susceptible d'être transformée et conduite d'une situation défavorable vers le bonheur, il unit l'ordre à la confusion désordonnée, la mesure à la démesure, la beauté à

187. Le substantif temeritas sert, chez Cicéron (Tusc. 2, 47), à désigner la partie irrationnelle de l'homme. On ne peut toutefois le rendre directement par irrationalité. Temeritas signifie « hasard aveugle, absence de calcul, irréflexion ». Ce dernier terme semble rendre l'idée du texte, le mot témérité étant trop fort parce qu'il implique l'idée d'une audace imprudente qui n'est peut-être pas à l'œuvre ici.

188. L'expression vise à associer les deux causes du Timée, l'intellect visant l'avènement du meilleur et la nécessité aveugle, avec les deux principes pythagoriciens : le dieu et la matière. On trouve un semblable rapprochement au début du traité du Pseudo-Timée de Locres.

189. Calcidius reprend le discours indirect, ici pour exprimer la pensée de Pythagore, bien entendu toujours par l'intermédiaire de Numénius. Le conditionnel vise ici encore à traduire le discours indirect.

190. Avec Dillon 1977, p. 375, et Moreschini 2003, p. 601, nous traduisons corporeorum comme un adjectif substantivé renvoyant à tout ce qui est corporel (la matière étant à l'origine du corporel), plutôt que comme un adjectif qualificatif se rapportant à deorum pour désigner les « dieux corporels », c'est-à-dire les astres. Toutefois, cette seconde traduction, plus traditionnelle (van Winden 1959, p. 115; Des Places 1973, p. 98 ; Bakhouche 2011, p. 527), est tout aussi valable. Le passage peut faire penser à celui où Xénocrate décrit la dyade comme la mère des dieux d'une part et comme l'âme du monde d'autre part (Aet. Plac. I, 7, 30, p. 304 Diels = fr. 15 Heinze = Fr. 213 Isnardi Parente) . 
atque exornando conuertit. Denique negat inueniri Numenius et recte negat inmunem [115] a uitiis usque quaque generatorum fortunam : non in artibus hominum, non in natura, non in corporibus animalium, nec uero in arboribus aut stirpibus, non in frugibus, non in aëris serie nec in aquae tractu, nec in ipso quidem caelo, ubique [120] miscente se prouidentiae deterioris naturae quasi quodam piaculo. Idemque nudam siluae imaginem demonstrare et uelut in lucem destituere studens detractis omnibus singillatim corporibus, quae gremio eius formas inuicem mutuantur et inuicem [125] mutant, ipsum illud, quod ex egestione uacuatum est, animo considerari iubet: eamque siluam et necessitatem cognominat. ex qua et deo mundi machinam constitisse deo persuadente, necessitate obsecundante. Haec est Pythagorae de originibus [130] adseueratio.

[Fin du fr. 52]

300. Superest ipsa nobis ad tractandum Platonis de silua sententia, quam diuerse interpretari uidentur auditores Platonis : quippe alii generatam dici ab eo putauerunt uerba quaedam potius quam rem secuti, alii uero sine generatione, sed anima praeditam, quando ante inlustrationem quoque motu instabili atque inordinato dixerit eam fluctuasse, cum motus intimus genuinusque sit uiuentium proprius: quodque idem la laideur et changea ainsi totalement son état en l'embellissant et en la parant. Enfin Numénius dit, et ce à juste titre, qu'on ne peut trouver, dans ce qui est engendré, une condition qui soit totalement exempte de défauts, ni dans les œuvres des hommes, dans la nature, dans les corps des animaux, ni dans les arbres ou les plantes, dans les fruits, dans le fil de l'air ou le cours de l'eau, ni même dans le ciel : partout se mêle à la providence comme une sorte de souillure qui appartient à la nature inférieure. Le même Numénius, s'appliquant à donner une image nue de la matière et pour ainsi dire à la mettre en lumière, prescrit de retirer un à un tous les corps qui, en son sein, s'empruntent et échangent réciproquement leurs formes, et de considérer en esprit cela même qui a été laissé après cette opération d'élimination ${ }^{191}:$ ce qu'il nomme à la fois matière et nécessité. C'est d'elle et du dieu que l'appareil du monde aurait été constitué, le dieu exerçant sa force persuasive, la nécessité lui obéissant. Voilà ce qu'affirme Pythagore à propos des origines.

$$
\text { [Fin du fr. 52] }
$$

300. Il nous reste à exposer la doctrine même de Platon sur la matière, que ses disciples ont manifestement interprétée de diverses façons. Les uns, en effet, ont cru que, selon lui, elle était engendrée, mais ils se sont plus fiés à certains mots qu'à ce qu'il voulait réellement dire ; d'autres ont pensé qu'il la considérait comme non engendrée, mais dotée d'une âme, puisqu'il avait dit qu'avant d'être embellie, elle était agitée d'un mouvement instable et désordonné et que le mouvement interne et inné est le propre des êtres vivants; c'est pourquoi aussi le même Platon avait affirmé qu'il existait deux âmes du 
saepe alias duas esse mundi animas dixerit, unam malignam ex silua, alteram beneficam ex deo. Existentibus itaque bonis ac malis bona quidem ex anima benefica mundo [329] tributa, incommoda porro ex siluestri maligna, cum diuina sapientia intelligentiaque opificis dei siluae seuere atque efficaciter persuaderet praebere cultui atque exornationi suae patientiam, persuasio uero non nisi animantibus uitaque fruentibus adhibeatur. Quibus Hebraei concinunt, cum dicunt homini quidem a deo datam esse animam ex inspiratione caelesti, quam rationem et animam rationabilem adpellant, mutis uero et agrestibus ex silua rationis monde distinctes, l'une, malfaisante, qui provenait de la matière, l'autre, bienfaisante, qui provenait du dieu. Ainsi, selon eux, comme les biens et les maux existent, les biens qui ont été accordés au monde sont issus ${ }^{192}$ de l'âme bienfaisante, tandis que les dommages lui sont venus de l'âme matérielle ${ }^{193}$ malfaisante, alors que la sagesse divine et l'intelligence du dieu démiurge s'efforçaient de persuader la matière, de manière dure, mais efficace, de se montrer docile et d'accepter ${ }^{194}$ les soins et la parure qu'il lui donnait. Or la persuasion ne peut être employée que pour les êtres qui sont doués d'une âme et jouissent de la vie ${ }^{195}$. Avec ces penseurs, les Hébreux sont d'accord, quand ils disent que l'âme a été donnée à l'homme par Dieu et qu'elle provient d'une inspiration céleste ${ }^{196}$ qu'ils appellent raison et âme rationnelle, tandis qu'aux animaux privés de paroles et vivant dans les champs ${ }^{197}$, en revanche,

192. Ici encore, le double ex est essentiel au sens. Avec tributa, comme avec datam au paragraphe 300, on aurait pu attendre un a pour désigner l'agent. Mais le texte spécifie ici l'origine des biens et des maux, et non l'agent qui les distribue.

193. Calcidius visiblement récrit l'exposé de Numénius qu'il vient de transcrire en latin (295-299) et qu'il considère comme une interprétation de Platon. En écrivant silvestris maligna, l'《âme matérielle mauvaise », il s'engage le moins possible sur la question de savoir si Numénius a parlé d'une âme de la matière ou d'une âme qui est matière (c'est pourquoi, en français, il faut garder l'ambiguïté et traduire silvestris par matérielle). En revanche, quand il rend compte ensuite de l'enseignement hébraiqque qu'il tire sans doute aussi de Numénius, il n'y a aucune hésitation sur le fait que l'âme irrationnelle provienne de la matière.

194. Littéralement, elle fait «montre de patience pour supporter» les soins du dieu. Patientia désignant toutefois moins la patience proprement dite que l'action de supporter, nous avons préféré rendre le terme par docile.

195. Le et a une fonction épexégétique. Calcidius explique ce que signifie être animé en disant que c'est posséder la vie. On pourrait aussi traduire par « et qui donc jouissent de la vie ». La double expression renvoie cependant en outre au vivis et animantibus de l'enseignement biblique qui suit et qu'elle sert à gloser par avance.

196. Ici la distinction est nette entre l'agent divin qui ordonne le don d'une âme et l'origine matérielle de cette âme exprimée par ex.

197. L'adjectif mutus désigne les bêtes (les « brutes ») comme étant privées de la parole (il est employé avec bestia et pecudes chez Cicéron, De finibus, I, 27, 71 et Epistulae ad Quintum fratrem, fr. 1, 1, 8, \$24). L'adjectif agrestis évoque peut-être quant à lui, plutôt que les animaux qui vivent dans les champs, plus généralement les animaux qui vivent sur terre (voir Cicéron, De amicitia, 81). Il sert en effet sans doute à rendre l'évocation des « tétrapodes » ou des « animaux de la terre » évoqués dans le verset biblique ici retranscrit (Gen. 
expertem iussu dei uiuis et animantibus bestiis terrae gremio profusis: quorum in numero fuerit etiam ille serpens, qui primitias generis bumani malis suasionibus inlaqueauerit.

a été donnée une âme dépourvue de raison qui provient de la matière, quand, sur l'ordre de Dieu, les bêtes vivantes et douées d'une âme ${ }^{198}$ sont sorties du sein de la terre ; dans leur nombre figurait aussi le fameux serpent qui, par ses mauvais conseils, a séduit les prémices du genre humain.

1,24 , voir la note suivante). Il est possible que Calcidius ait tenté de rendre par ces deux

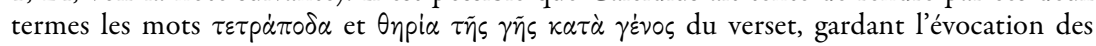
$\varepsilon p \pi \varepsilon \tau \dot{\alpha}$ pour la fin du passage où il mentionne le serpent. En outre, au sens figuré, ces deux adjectifs servent à désigner des individus grossiers et donc privés de raison, ce qui est exactement le propos philosophique à l'œuvre.

198. Le latin (comme sans doute le grec de Numénius avant lui) tend à rendre ici le propos de Gen. 1, 24 où sont juxtaposées les notions d'âme et de vie dans l'expression $\dot{\eta} \gamma \tilde{\eta}$

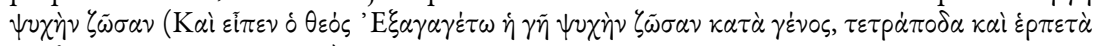

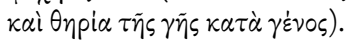




\section{BIBLIOGRAPHIE}

Bakhouche, B. 2011 : Calcidius, Commentaire au Timée de Platon, édition critique, traduction française, notes sur la traduction et le Commentaire de Calcidius et annexes, Paris, 2011 (Histoire des doctrines de l'Antiquité classique, 42).

BALTES, M. 1975: «Numenios von Apamea und der platonische Timaios », Vigiliae christianae, 29 (1975), p. 241-270 (= A. Hüffmeier, M.-L. Lakmann \& M. Vorwerk [éd.], $\triangle I A N O H M A T A$ : Kleine Schriften zu Platon und zum Platonismus, Leipzig, 1999 [Beiträge zur Altertumskunde, 123], p. 1-32).

BonazzI, M. 2013: «Pythagoreanizing Aristotle : Eudorus and the Systematization of Platonism », dans Schofield 2013.

BRISSON, L. [1974] ${ }^{3} 1998$ : Le Même et l'Autre dans la structure ontologique du Timée de Platon: un commentaire systématique du Timée de Platon, $3^{c}$ édition, revue, pourvue de corrigenda, d'addenda, d'index révisés et surtout d'une bibliographie analytique nouvelle mise à jour, Sankt Augustin, ${ }^{3} 1998$ (International Plato studies, 2).

- 2000 : « Aristote, De anima, I 2, 404b16-30 », dans Id. Lectures de Platon, Paris, 2000 (Bibliothèque d'histoire de la philosophie), p. 90-98. [Première version dans Les Études philosophiques, 1990/1, p. 95-105.]

- 2011: «The Mortal Parts of the Soul, or Death as Forgetting the Body », dans M. Migliori, L. M. Napolitano Valditara, A. Fermani (éd.), Inner Life and Soul: Psychè in Plato, Sankt Augustin, 2011 (Lecturae Platonis, 7), p. 63-70.

BuRnet, J. [1914] 1957 : Early Greek Philosophy, New York, ${ }^{4} 1957$ (Meridian Library, 5).

Cherniss, H. 1993: L'énigme de l'ancienne Académie, introduction et traduction de L. Boulakia. Suivi en appendice de : E. E. N. Tigerstedt, Le système caché, Paris, 1993 (Tradition de la pensée classique) [traduction de The Riddle of the Early Academy, Berkeley, 1945].

- 1976: Plutarch, Moralia, in Seventeen Volumes. 13.1, with an English Translation, Cambridge (Mass.)-Londres, 1976 (The Loeb Classical Library, 427).

CHIARADONNA, R. 2011 : « Interpretazione filosofica e ricezione del corpus : il caso di Aristotele $(100$ a. C. -250 d. C.) », dans L. Del Corso, P. Pecere (éd.), Il libro filosofico dall'Antichità al XXI secolo/Philosophy and the Books: From Antiquity to the $21^{\text {th }}$ Century = Quaestio, 11 (2011), p. 83-114.

- 2013: «Platonist Approaches to Aristotle : From Antiochus of Aschalon to Eudorus of Alexandria (and beyond) », dans Schofield 2013, p. 28-52.

CORNFORD, F. M. 1937 : Plato's Cosmology : the Timaeus of Plato, translated with a running commentary, Londres, 1937 (International Library of Psychology, Philosophy and Scientific Method).

DeCleva CaizZI, F. 1988: «La "materia scorrevole" : sulle tracce di un dibattito perduto », dans J. Barnes \& M. Mignucci (éd.), Matter and Metaphysics: Fourth Symposium Hellenisticum, Naples (Elenchos, 14), p. 425-470.

Des PlaCeS, É. 1973 : Numénius, Fragments, texte établi et traduit par —, Paris, 1973 (Collection des Universités de France).

DodDS, E. R. [1933] 1963: Proclus, The Elements of Theology, a revised text with translation, introduction and commentary, Oxford, ${ }^{2} 1963$.

Donini, P. L. 1982 : Le scuole, l'anima, l'impero : la filosofia antica da Antioco a Plotino, Turin, 1982 (Sintesi, 3).

- 1992: «Plutarco e i metodi dell'esegesi filosofica », dans I. Gallo \& R. Laurenti (éd.), I Moralia di Plutarco tra filologia e filosofia : atti della giornata plutarchea di Napoli, Istituto Suor Orsola Benincasa, Naples, 1992 (Strumenti per la ricerca plutarchea, 1), p. 79-96. 
- 2004: «Plutarco e Aristotele », dans I. Gallo, La biblioteca di Plutarco : atti del IX Convegno plutarcheo, Pavia, 13-15 giugno 2002, Naples (Collectanea, 23), p. 255273.

- 2011 : «Medioplatonismo e filosofi medioplatonici : una raccolta di studi », dans Id., Commentary and Tradition: Aristotelianism, Platonism and Post-Hellenistic Philosophy, ed. by M. Bonazzi, Berlin-New York, 2011 (Commentaria in Aristotelem Graeca et Byzantina, 4), p. 283-296. [=Elenchos 11 (1990), p. 79-93.]

DÖRRIE, H. \& M. BALTES 1996 : Der Platonismus in der Antike: Grundlagen, System, Entwicklung, 4: Die philosophische Lehre des Platonismus: einige grundlegende Axiome, platonische Physik (im antiken Verständnis). 1 : Bausteine 101-124: Text, Übersetzung, Kommentar, Stuttgart-Bad Cannstatt, 1996.

EDWards, M. J. 2010 : « Numenius of Apamea », dans L. P. Gerson (éd.), The Cambridge History of Philosophy in Late Antiquity, chap. 6, Cambridge, p. 115-125.

FinAmore, J. F. \& J. M. Dillon 2002 : Iamblichus, De anima, text, translation, and commentary, Leyde-Boston-Cologne, 2002 (Philosophia antiqua, 92).

Ferrari, Fr. 2007 : « La chora nel Timeo di Platone : riflessioni su "materia" e "spazio" nell' ontologia del mondo fenomenico », Quaestio, 7 (2007), p. 3-23.

Ferrari, Fr. \& L. BALDI 2002 : Corpus Plutarchi Moralium, 37 : La Generazione dell'anima nel Timeo, introduzione, testo critico, traduzione e commento, Naples, 2002.

Frede, M. 1987 : art. « Numenius », dans W. Haase (éd.), Aufstieg und Niedergang der römischen Welt (ANRW), II.36.2, Berlin, 1987, p. 1034-1075.

HageR, Fr.-P. 1987 : Gott und das Böse im antiken Platonismus, Amsterdam-Würzburg, 1987 (Elementa : Schriften zur Philosophie und ihrer Problemgeschichte, 43).

Happ, H. 1971 : Hyle: Studien zum Aristotelischen Materie-Begriff, Berlin-New York, 1971.

HeinZe, R. 1892: Xenokrates: Darstellung der Lehre und Sammlung der Fragmente, Leipzig, 1892.

Helleman-Elgersma, W. 1980 : Soul-Sisters : a Commentary on Enneads IV 3 [27], 1-8 of Plotinus, Amsterdam-Hildesheim, 1980 (Elementa : Schriften zur Philosophie und ihrer Problemgeschichte, 15).

ISAAC, D. 1982 : Proclus, Trois études sur la Providence. 3 : De l'existence du mal, texte établi et traduit par - , avec une note additionnelle de C. Steel, Paris, 1982 (Collection des Universités de France).

IsNard PARENTE, M. 1982: Senocrate, Ermodoro, Frammenti, edizione, traduzione e commento, Naples, 1982 (La scuola di Platone, 3).

Johansen, Th. Kj. 2004: Plato's Natural Philosophy: a Study of the Timaeus-Critias, Cambridge-New York, 2004.

JOURDAN, F. 2013a-b : « La matière à l'origine du mal chez Numénius : un enseignement explicité chez Macrobe ? », Revue de philosophie ancienne, 31/1 (2013), p. 41-98; 31/2 (2013), p. 149-178.

-2013c : «Le fragment 43 Des Places de Numénius : problèmes de présentation, essais d'interprétation », Les Études platoniciennes, 10 (2013) (http://etudes platoniciennes.revues.org/321)

— 2014: « Materie und Seele in Numenios' Lehre vom Übel und Bösen », dans F. Jourdan \& R. Hirsch-Luipold (éd.), Die Wurzel allen Übels: Vorstellungen über die Herkunft des Bösen und Schlechten in der Philosophie und Religion des 1.-4. Jahrbunderts [Actes du colloque international organisé au Lichtenberg-Kolleg, 2729 Janvier 2011], Tübingen (Ratio Religionis Studien, 3).

- 2015: «Eusèbe de Césarée et les extraits de Numénius dans la Préparation évangélique », dans S. Morlet (éd.), Actes du colloque « Lire en extraits » (30 nov$1^{\text {er }}$ déc. 2012), Paris (à paraître). 
KeHL, A. 1978 : Art. « Gewand (der Seele)», dans Th. Klauser, C. Colpe \& E. Dassmann et al. (éd.), Reallexikon für Antike und Christentum: Sachwörterbuch zur Auseinandersetzung des Christentums mit des antiken Welt, 10, Stuttgart, 1978, col. 9451025.

MenN, St. 1995 : Platon on God as Nous, Carbondale (Ill.), 1995 (The Journal of the History of Philosophy. Monograph Series).

- 1998 : Descartes and Augustine, Cambridge, 1998.

Moreschini, C. 2003 (éd.) : Calcidio, Commentario al Timeo di Platone, Milan, 2003 (Il pensiero occidentale).

Plese, Z. 2005 : «Platonist Orientalism », dans A. Pérez Jiménez \& F. Titchener (éd.), Historical and Biographical Values of Plutarch's Works: Studies devoted to Professor Philip A. Stadter by the International Plutarch Society, Málaga-Logan (Utah), p. 355-382.

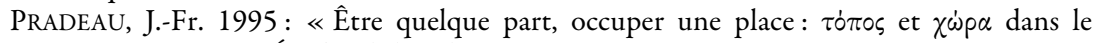
Timée », Les Études philosophiques, 1995/3, p. 375-399.

PueCH, H.-Ch. 1934: « Numénius d'Apamée et les théologies orientales au second siècle », Annuaire de l'institut de philologie et d'histoire orientales, 2 (1934) = Mélanges Bidez, p. 745-778.

REALE, G. 1984: Per una nuova interpretazione di Platone: rilettura della metafisica dei grandi dialoghi alle luce delle "dottrine non scritte", Milan, ${ }^{5}$ 1987, [con in appendice un saggio di H. Krämer sul presente volume] (Centro di ricerche di metafisica. Sezione di metafisica del platonismo nel suo sviluppo storico e nella filosofia patristica. Studi e testi, 3).

Richard, M. D. [1986] 2005: L'enseignement oral de Platon, Paris, 1986, éd. revue et corrigée, 2005.

Schofield, M. 2013 : Aristotle, Plato and Pythagoreanism in the First Century BC, Cambridge, 2013.

SwitAlski, B. W. 1902 : Des Chalcidius Kommentar zu Plato's Timaeus : eine historischkritische Untersuchung, Münster, 1902 (Beiträge zur Geschichte der Philosophie des Mittelalters, 3.6).

TAYLOR, A. E. 1928 : A Commentary on Plato's Timaeus, Oxford, 1928.

THEILER, W. : 1957 : « Gott und Seele im kaiserzeitlichen Denken », dans Recherches sur la tradition platonicienne, Vandœuvres-Genève, 1957 (Entretiens sur l'Antiquité classique, 3), p. 65-91 (= Id. Forschungen zum Neuplatonismus, Berlin, 1966, p. 104123).

VAN WINDEN, J. C. M. 1959, Calcidius on Matter: his Doctrine and Sources. A Chapter in the History of Platonism, Leiden, 1965 (Philosophia antiqua, 9). [Réimpr. 1965.]

WasZINK, J.H. 1962 : Plato latinus. 4, Timaeus a Calcidio translatus commentarioque instructus, Londres-Leyde, 1962 (Corpus philosophorum medii aevi. Corpus Platonicum).

— 1966, « Porphyrios und Numenios », dans Porphyre, Vandœuvres-Genève, 1966 (Entretiens sur l'Antiquité classique, 12), p. 33-83.

Zambon, M. 2002 : Porphyre et le moyen-platonisme, Paris, 2002 (Histoire des doctrines de l'Antiquité classique, 27). 